\title{
Logical Combinatorialism
}

\author{
Andrew Bacon*
}

January 1, 2020

\begin{abstract}
In explaining the notion of a fundamental property or relation, metaphysicians will often draw an analogy with languages. The fundamental properties and relations stand to reality as the primitive predicates and relations stand to a language: the smallest set of vocabulary God would need in order to write the 'book of the world'. In this paper I attempt to make good on this metaphor. In order to do this I introduce a modality that, put informally, stands to propositions as logical truth stands to sentences. The resulting theory, formulated in higher-order logic, also vindicates the Humean idea that fundamental properties and relations are freely recombinable and a variant of the structural idea that propositions can be decomposed into their fundamental constituents via logical operations. Indeed, it is seen that, although these ideas are seemingly distinct, they are not independent, and fall out of a natural and general theory about the granularity of reality.
\end{abstract}

Metaphysicians often theorize in terms of the notion of a property or relation being fundamental. When it comes to spelling out what this means, metaphors abound. According to one gloss, the fundamental properties and relations are the primitive constants in the language of reality, from which all other properties and relations can be defined; they are the vocabulary God would need in order to write the 'book of the world' (Sider (2011)).

The metaphor of reality as a language is one that is hard to make precise, and, taken too literally, has been shown to be subject to paradoxes. ${ }^{1}$ I take this as a warning to proceed cautiously, by codifying our metaphysics in a precise formal language where possible — in this case, higher-order logic — and employing model theoretic techniques to check for consistency. The appropriate starting point for our investigation turns out to be with an examination of the notion of logical possibility: a modality that, putting it informally, stands to reality as logical consistency stands to language. This is introduced and developed in section 1 and 2. We begin our examination of the language-reality analogy in section 3 by exploring one way in which the fundamental might be like the primitive non-logical expressions of a language: they are modally malleable. They freely vary their structural relations across modal space in the same way the primitive expressions

\footnotetext{
${ }^{*}$ Thanks are due to many people. Thanks to Jan Plate, for catching several infelicities, Michael Caie, Peter Fritz, Lloyd Humberstone and Harvey Lederman for reading a lengthy earlier draft of this paper and providing many helpful thoughts, and to John Hawthorne and Jeff Russell for many discussions of related issues and their encouragement with the overall project. Special thanks are due to Cian Dorr and Jeremy Goodman, who have provided invaluable feedback that has shaped much of my thinking on these issues. I'd particularly like to thank the referee for this journal, who forced me, to the great benefit of the paper, to make it far less technical, and shorten it by about twenty pages, as well as suggesting several substantive improvements. Many thanks, also, to Catrin Campbell-Moore for generously creating the diagram in the appendix.

${ }^{1}$ The problem is an old one (see Russell (1937) Appendix B, Myhill (1958)), but the moral hasn't been taken to heart among metaphysicians until quite recently. See Uzquiano (2015), Dorr (2016), Goodman (2017).
} 
(but not the defined expressions) may freely vary their interpretations across models of a language. These recombinatorial principles fall directly out of our theory of logical possibility, and unify and systematize a number of extant principles in the literature intended to secure the Humean idea that there are no necessary connections between the fundamental properties and relations.

In section 4 , I argue that our broadly Humean theory of fundamentality and possibility is able to make good on a substantially larger fragment of the language-reality metaphor than has been previously recognized. The theory vindicates the thesis that propositions, properties and relations may be decomposed into their fundamental components via logical operations, and ensures that this decomposition is unique modulo certain structural manipulations (intended to allow, for instance, a proposition to be multiply decomposed as a relation applied to its arguments in one order, or its converse in the other). We also obtain the principle that the fundamental are simple, in the sense that they cannot be decomposed into other fundamental properties and relations. In section 5, I briefly explain how to model our combinatorial theory of possibility in a way that elucidates the connection between logical possibility and fundamentality. I conclude in section 6 .

The theory proposed here is strong, and settles many contentious questions of metaphysics: it tells us, for instance, that there are no symmetric fundamental relations, and that the fundamental properties and relations can exhibit arbitrary patterns of instantiation. Rather than approaching such questions in a piecemeal way, as is representative of these sorts of investigations, I have advanced a single principle which decides these questions all at once. ${ }^{2}$ I think this sort of predictive strength should not be held against the theory: debates in these areas of metaphysics are often quite unconstrained, and it is a virtue when a single coherent picture of reality answers the central questions uniformly. The resultant theory should be adjudicated by the usual standards of philosophical theorizing: according to the merits of strength, simplicity, consistency with the sciences, predictive ability, and so forth.

Many of the ideas in this paper can be modeled in a more general formal framework for thinking about metaphysical structure outlined in Bacon (forthcoming). That paper may be seen as a technical companion to this one; here I proceed comparatively informally. The reader wanting to look into the relevant technical tools can find further details in that paper, and the appendix to this one.

\footnotetext{
${ }^{2}$ There is certainly incentive to approach such questions individually, for after all, a strong theory that resolves many questions is open to a greater number of objections than could be made by resolving any given question individually. But simply tallying objections is evidently not a methodologically sound way to proceed, insofar as it recommends withholding judgment over taking stances on particular matters. Indeed, the piecemeal approach has its own distinctive drawbacks. Considerations that bear more weight in one area of metaphysics, bear less in another. Often this is for purely sociological reasons. Sometimes it is a hard earned philosophical lesson of the dispute in question that certain considerations must take a backseat, one that is not always carried over to other debates. This is clearly not to say that one should ignore the local consequences of a strong theory when evaluating it: these considerations do suggest, however, that one should temper the instinct to remain neutral on contentious matters.
} 


\section{Logical Consistency}

In what follows we will explore the Humean idea that the fundamental properties and relations can occupy any logically consistent role. But this in itself requires some explanation, for in its customary sense logical consistency is a predicate of sentences of some particular language, not of propositions built out of properties and relations. Informally, a sentence is logically consistent if it is true under some interpretation of the primitive non-logical constants. In advancing our analogy between language and reality, we shall introduce a propositional modality that stands to reality as logical consistency stands to language, where, in this analogy, fundamental properties and relations play the role of the primitive non-logical constants. With this idea properly set forth, we will be able to articulate various more substantive theses about the structure of reality. In this section we'll begin by briefly examining the conventional notion of logical truth, and its dual, logical consistency, as it applies to language.

We do not want to limit our investigation to the hypothesis that only individuals, properties and relations can be fundamental. If tense or modal operators are candidates for being among the fundamental, then we must take seriously the idea that operators are among the fundamental. Sider (2011) similarly takes up the question of whether quantifiers are fundamental. In order to be impartial on these matters, we will not make any assumptions about which types the fundamental occupy. To be able to express suitably general claims we also need to be able to quantify into the position that a name, predicate, operator, and so forth, can occupy, making the appropriate framework for our investigation higher-order logic. A higherorder language may contain non-logical constants belonging to different grammatical categories, including the familiar categories of first-order logic, which has individual and predicate constants, but also categories corresponding to sentences, operators, and many others. These grammatical categories are encapsulated by a system of types: type $e$ and $t$ correspond to the category of singular terms and sentences respectively, and the type $\sigma \rightarrow \tau$ is the category of expressions that combine with something of type $\sigma$ to produce something of type $\tau$; for instance, an operator combines with a sentence to form a sentence, and thus has type $t \rightarrow t$. A higher-order language also contains, for any given type, variables that can take the position of expressions of that type, and quantifiers that can bind them. The language also contains a variable binding symbol, $\lambda$, for making predicates out of open formulae, and doing analogous things to open expressions of other types (the function of this device will become clearer as we proceed). An exact specification of this language may be found in appendix 7.1; in the text we shall proceed informally, providing details as necessary.

Let's begin by examining the conventional notion of logical truth, and its dual, logical consistency, for higher-order languages. According to one popular conception, attributed to Bolzano, a logical truth is a sentence that is true in virtue of the logical form of the sentence alone. Thus for example, the sentence 'if 
John is tall then John is tall' is a logical truth because every sentence with the same logical form as it is true: 'if Jane is short then Jane is short', 'if Jane likes Mary then Jane likes Mary', and so on. On the other hand, 'John is tall' is not a logical truth because there are false sentences with the same logical form; assuming John is in fact tall, an example would be 'John is short'. One way to make this idea precise is to appeal to the notion of a substitution of the language: a mapping from sentences to sentences that replaces some of the non-logical constants with things of the same syntactic type (e.g. replacing 'tall' with 'short', and so on, but fixing logical constants like 'if' and 'not'). A sentence is a logical truth if and only if all of its substitution instances are true. And it is logically consistent if and only if it has a true substitution instance.

This analysis is instructive because it brings out a formal analogy, that we will exploit later, with the possible worlds analysis of metaphysical possibility, according to which a proposition is metaphysically necessary (possible) iff it is true at all (some) possible worlds. Substitutions thus stand to logical modality as possible worlds stand to metaphysical modality. However, the drawback of the substitutional analysis, at least as it presently stands, is that it is language dependent in an objectionable way. Impoverished languages have fewer substitutions. A counterexample to the logical truth of a sentence might be available in a more expressive extension of that language, so that a single sentence might be a logical truth relative to one language, but not another, even when the meaning of the sentence is the same in both. ${ }^{3}$

It will thus be convenient, for the time being, to consider another more semantic definition of logical truth, due to Tarski. Our second parse becomes: ${ }^{4}$

A sentence $A\left(c_{1} \ldots c_{n}\right)$ is a logical truth iff $\forall x_{1} \ldots x_{n} A\left(x_{1} \ldots x_{n}\right)$ is true.

Dually, we will say that $A\left(c_{1} \ldots c_{n}\right)$ is logically consistent iff $\exists x_{1} \ldots x_{n} A\left(x_{1} \ldots x_{n}\right)$ is true. This definition requires a little explanation. In the above $A\left(c_{1} \ldots c_{n}\right)$ represents a sentence containing non-logical constants $c_{1} \ldots c_{n}$, and $A\left(x_{1} \ldots x_{n}\right)$ represents the sentence one obtains from $A$ by substituting each constant $c_{i}$ with a variable $x_{i}$ of the same type. Note that in the right-hand-side we are making use of the fact that our language has quantifiers that allow us to quantify into any grammatical position. Thus, for example, the sentence 'if John is tall then John is tall' is a logical truth roughly because the higher-order sentence 'for any property $X$ and any individual $y$, if $y$ is $X$ then $y$ is $X^{\prime}$ is true. ${ }^{5}$ In what follows we will adopt the convention of writing $\bar{c}$ as short for the sequence of constants $c_{1} \ldots c_{n}$ and $\bar{x}$ for $x_{1} \ldots x_{n}$. We will refer to this conception of logical truth as the Bolzano-Tarski account logical truth, although we follow Tarski in

\footnotetext{
${ }^{3}$ However, see Halbach (forthcoming), for a more sophisticated development of the substitutional analysis of logical truth.

${ }^{4}$ See Tarski (1983). Although this way of formulating logical truth is not explicit in Tarski's writing, it appears in some discussions by later logicians; see, for example, in Kreisel (1962). This definition of logical truth also plays a big role in Williamson (2013) (see the discussion of 'metaphysical universality').

${ }^{5}$ Above, and throughout this paper, I will adopt a natural paraphrase of higher-order logic into English where one approximates quantification into predicate position with singular talk of properties, quantification into sentence position with singular talk of propositions, and so on. The intended sentence of higher-order logic is always easily recoverable from the paraphrase, although they sometimes require one to take some liberties with grammar.
} 
defining it semantically.

The definition of logical truth may look a little alien to the modern reader since contemporary Tarskian accounts of logical validity are presented in model-theoretic terms. A sentence is logically true if it is true under every interpretation of the non-logical vocabulary, where interpretations are set-theoretic constructions. However, Tarski originally provided his definition of truth and logical truth in a higher-order language, where quantification over possible interpretations for the predicates was achieved by using quantification into predicate position, and similarly for other grammatical categories. Indeed, there are good reasons to prefer this approach (see Williamson (2003)). ${ }^{6}$

\section{Logical Possibility}

In this section I will outline a theory that, I contend, encapsulates a substantial fragment of the coveted language-reality correspondence cited in the introduction. The key idea is to posit a modality, $\diamond$, governed by a basic postulate stating, roughly, that it stands to reality as logical consistency stands to language. We will use this theory in later sections to explore the recombinatorialist idea that logical consistency is a guide to possibility, and to derive several theses about the structure of properties and relations.

The Tarskian notion of logical consistency, as we have seen, is one that applies to sentences: linguistic entities that are structured in accordance with the order in which they are built up out of constants and the basic syntactic operations. Logical consistency merely describes features of our representations of the world. By contrast, a propositional modality, such as metaphysical possibility, directly describes non-representational features of the world. ${ }^{7}$ It is consequently a substantive thesis that propositions be sufficiently similar to sentences as to admit a corresponding notion of consistency. (To dramatize the issue, observe that while the property of containing three vowels carves a non-trivial distinction between sentences, it evidently has no interesting analogue in reality, since propositions presumably aren't sentence-like enough to contain vowels.)

People sometimes talk about logical necessity — conceived as a propositional modality — as though it is the broadest kind of necessity: that any proposition that is possible in any given (propositional) sense

\footnotetext{
${ }^{6}$ See also Rayo and Williamson (2003), Williamson (2000), Rayo and Uzquiano (1999). The above notion of logical truth is one in which we do not vary the interpretation, and in particular the domain, of the universal quantifiers, since they are logical constants. For example, since we can say 'there are at least five things' without using non-logical constants, this sentence will be a logical truth provided it is in fact true. This is also in accordance with Bolzano's substitutional analysis, and Tarski's original definition of logical truth, and is a feature that has been embraced by some authors, most prominently by Timothy Williamson. See Williamson (2013), Williamson (2000), Rayo and Williamson (2003). (See Etchemendy (1990) for some critical discussion. I take this dispute to be partly terminological; I would happily reserve the word 'logic' for what is knowable $a$ priori or metaphysically necessary, but the notion I have identified above would still exist and be worthy of study. One could even understand logical truth as the conjunction of all three ideas, although it is unclear to me whether such a concept would be useful.) There is an alternative conception of logical truth, adopted in Tarski's later works, in which the domain of the quantifiers is allowed to vary along with the non-logical constants. Although we won't pursue the variant notion here, the above definition can be modified to accommodate it.

${ }^{7}$ Pace many early and mid-twentieth philosophers, such as Carnap and Quine.
} 
of possibility - physical, metaphysical, or what have you - must automatically be logically possible. In higher-order logic one can actually prove, given some substantive, but reasonable assumptions (more on this shortly), that there is a broadest necessity. One way to define it is: being identical to $\top$, or $\lambda p$. $(p=\top)$, where T stands for a tautology. ${ }^{8}$ But higher-order logic is entirely neutral about the behavior of this operator, except that it is governed by a modal logic containing at least the theorems of S4. ${ }^{9}$ For instance, higherorder logic is consistent with a Fregean picture in which there are only two propositions, and the broadest necessity is a truth functional operator according to which there is no contingency. A modality that stood to reality as logical consistency stands to language, by contrast, would classify some propositions as being highly contingent: those corresponding, under the analogy with language, to logically contingent sentences. ${ }^{10}$

A sharper objective, then, is to formulate a thesis that forces $\square$, understood now as the broadest necessity, to behave in a way analogous to the way that logical truth behaves with respect to language. In a slogan, that broad necessity stands to reality as logical truth stands to language. In order to make this slogan precise, we need to tackle another question. Certain expressions - the primitive non-logical constants play a distinguished role in the definitions of logical truth and consistency, for it is only they which are available for substitution in the Bolzano definition, and that are quantified out in the Tarskian definition. One would expect a similarly distinguished collection of properties and relations to make an appearance in the analogous explication of a notion of logical necessity and possibility. What, under this analogy between language and reality, corresponds to the primitive constants of a language?

In what follows we shall make some attempts to characterize the idea that certain properties, relations, operators, and so on, are metaphysically simple or fundamental. According to a widespread, albeit nebulous metaphor, the fundamental properties and relations are those from which arbitrary properties and relations are built. This is supposed to be, in some respects (but not all!), analogous to the way that an arbitrary expression of a language can be built out of the primitive constants of that language via the structural rules and background logical vocabulary. If reality is God's language, then the fundamental entities are the primitive (non-logical) constants of that language. It turns out that the notion of propositional logical necessity and the resultant notion of fundamentality are intimately entwined, and cannot be characterized independently.

Let us suppose, then, that $\mathcal{L}$ is a higher-order language and that it is moreover a fundamental language: on

\footnotetext{
${ }^{8}$ The assumptions in question ensure that it does not matter which tautology is used. This operator has several equivalent formulations. An alternative that makes its role as the broadest necessity especially obivous is the definition: being a $p$ such that every necessity applies to $p$ (that is, $\lambda p \forall X(N e c(X) \rightarrow X p)$ ). The assumptions, and the definitions required to make this precise - including a higher-order definition of being a necessity operator (formalised Nec, above) — are described in Bacon (2018a).

${ }^{9}$ Indeed, it is consistent that its exact logic is any modal logic characterized by a single transitive reflexive frame, such as S4 or S5. See Bacon (2018a) for more details.

${ }^{10}$ For example, $\exists x F x$, where $F$ is a non-logical predicate constant is both logically consistent and has a logically consistent negation.
} 
its intended interpretation, the constants denote fundamental entities, and there is moreover no redundancy — no two constants denote the same fundamental entity. ${ }^{11}$ (Recall that a constant, in the context of higherorder logic, may belong anywhere in the type hierarchy, so we may assume as we please that our fundamental constants include predicates, operators, quantifiers, and so on.) In order to spell out what it means for a propositional operator to stand to reality as logical truth stands to language, we might follow the BolzanoTarski definition - that the sentence $A(\bar{c})$ is logically true iff $\forall \bar{x} A(\bar{x})$ is true simpliciter — and postulate that broad necessity satisfy the schema:

\section{Logical Necessity $\square A(\bar{c}) \leftrightarrow \forall \bar{x} A(\bar{x})$.}

where $A(\bar{c})$ is a sentence of $\mathcal{L}$ containing the non-logical constants $\bar{c}$. Notice that, unlike our characterization of logical truth, Logical Necessity is not a definition, where the notion of logical truth being defined resides in a different language to the language for which it's being defined. Logical Necessity is an object-language biconditional; the right-hand-side cannot be the definiens as $A$ may contain the operator $\square$ itself. At any rate, since we have already defined $\square$ as $\lambda p .(p=\top)$, Logical Necessity should be thought of as a substantive thesis about the behaviour of that operator.

Logical Necessity is equivalent to a dualized version of itself, $\diamond A(\bar{c}) \leftrightarrow \exists \bar{x} A(\bar{x})$, and we shall regularly appeal to these interchangeably. While Logical Necessity is helpful for getting a fix on the idea of what a notion of logical necessity must look like, it is possible to refine it in several directions.

We shall see shortly that Logical Necessity guarantees that the entities denoted by non-logical constants are extremely modally malleable: that every logical role that's in fact occupied could be occupied by the denotations of the constants. For example, take the property of being a truthful operator: ${ }^{12} \lambda X \forall p(X p \rightarrow p)$, and consider a non-logical operator constant $N$. Since there are truthful operators $-\exists X \forall p(X p \rightarrow p)-$ it follows by Logical Necessity that $N$ is possibly truthful $-\diamond \forall p(N p \rightarrow p)$.

Note that this means that no non-logical constant in our language can denote negation since one can prove that negation is necessarily not truthful. ${ }^{13}$ The modal malleability of the denotations of the nonlogical constants is analogous to the way that in model theory the interpretation of a non-logical constant is unconstrained. At the other extreme the interpretation of logical constants, such as negation, cannot vary between models. And in between these extremes we have expressions built out of both logical and non-logical constants whose interpretations can only vary subject to constraints. (For instance the interpretation of round and not red can vary between models, but its interpretation is completely determined by the interpretation of

\footnotetext{
${ }^{11} \mathrm{~A}$ formal definition of a fundamental language, with respect to a given model of reality, can be found in the technical companion paper Bacon (forthcoming).

${ }^{12}$ Note that factive operators are truthful. The terminology of 'factive operators' often brings with it the implication that the operator be (at least) metaphysically necessarily truthful.

${ }^{13}$ We can evidently derive $\neg \forall p(\neg p \rightarrow p)$. Moreover, since this sentence contains no non-logical constants, it is equivalent to its own necessity by a vacuous instance of Logical Necessity.
} 
round and red.) This means that the restriction of Logical Necessity to instances in a fundamental language is important. ${ }^{14}$ This also makes it a somewhat peculiar schema: it is not a schema that would necessarily continue to hold if we extended the language with new constants denoting new things.

In this sense, Logical Necessity is as much an elucidation of fundamentality as the notion of logical necessity. One way to directly axiomatize this notion of fundamentality, whilst avoiding the restrictive assumption that we are theorizing in a fundamental language, is to introduce a class of predicates, $F u n^{\sigma}$, into the language, where $F u n^{\sigma}(a)$ expresses the idea that $a$ is one of the fundamental or metaphysically simple things of type $\sigma$. We shall adopt the convention of writing $\bar{x}$ or $\bar{c}$ as short for a sequence of variables $x_{1} \ldots x_{n}$ or constants $c_{1} \ldots c_{n}$. If $\bar{x}$ is a sequence of variables of type $\sigma_{1} \ldots \sigma_{n}$, it is useful to introduce a shorthand for the conjunction of claims stating that, for each $i, x_{i}$ is fundamental ${ }^{\sigma_{i}}$, and that any two $x_{i}$ of the same type are distinct. We will write this simply as $F u n(\bar{x})$, where the relevant conjunction can be inferred from the types of the variables $x_{1} \ldots x_{n} \cdot{ }^{15}$

We could now restate Logical Necessity as follows, without making any assumption about the constants of the language:

$$
\forall \bar{z}(F u n(\bar{z}) \rightarrow(\square A(\bar{z}) \leftrightarrow \forall \bar{x} A(\bar{x}))
$$

Here $A$ is a sentence in variables $\bar{x}$ that contains only logical constants. The idea here is that instead of the requirement that the constants denote fundamental entities, we universally quantify over fundamental things, and instead of the no redundancy requirement - that the constants do not codenote — we just require that the fundamental entities be pairwise distinct (the constraint implicit in our shorthand Fun $(\bar{z})$ ).

Finally, we have restricted $A(\bar{x})$ to formulas stated using only logical vocabulary: $\lambda \mathrm{s}$, variable, quantifiers and truth functional connectives. But one might wish to have a wider conception of which operations are logical, perhaps one that outstrips those expressible from the short list I mentioned above. ${ }^{16}$ Thus we could introduce a predicate, which I shall write $\operatorname{Pure}^{\sigma}(a)$, that says that $a$ is a something that is logical in the preferred sense. The final version is thus: ${ }^{17}$

\footnotetext{
${ }^{14}$ The constraint on fundamental languages that distinct constants do not denote the same fundamental entities is not idle either. For example, suppose that Venus is simple, and is denoted by two names $h$ and $p$ ('Hesperus' and 'Phosphorus'). Given Leibniz's law, we can infer $\square h=p$ from $h=p$ and $\square h=h$. Given $\square h=p$ Logical Necessity entails the absurd claim that there is at most one thing: $\forall x \forall y x=y$. Together, these observations mean the correlation between constants and denoted metaphysically simple entities must be one-to-one.

${ }^{15} \mathrm{So}$, for instance, if $x$ and $y$, have type $e$ and $Z$ type $e \rightarrow t$, then $F u n(x y Z)$ is short for $\operatorname{Fun}^{e}(x) \wedge F_{u n} e(y) \wedge F u n^{e \rightarrow t}(Z) \wedge x \neq$ $y$.

${ }^{16}$ I have my own theory about what these are, explained in Bacon (forthcoming) and section 5. Tarski offered another popular account: those operations that are invariant under permutations of the domain of individuals (see Tarski (1986)). Both outstrip the operations that are expressible from the list of logical operations I listed above. But there are other options as well (see, e.g., Feferman (2015)).

${ }^{17}$ Note that it is also possible to strengthen Quantified Logical Necessity by prefixing it with another $\square$. The original principle Logical Necessity cannot be obviously necessitated in the same way. For suppose that it was possible that $\bar{c}$ are not simple entities even though they in fact are (we shall later see that this must indeed be the case according to our conception of logical necessity). At such possibilities $\square A(\bar{c})$ may fail to be equivalent to $\forall \bar{x} A(\bar{x})$ for the same reasons they fail when $\bar{c}$ fail to denote simple entities. Quantified Logical Necessity quantifies over whatever entities happen to be the fundamental entities at
} 


\section{Quantified Logical Necessity $\forall X \forall \bar{z}(\operatorname{Pure}(X) \wedge F u n(\bar{z}) \rightarrow(\square X(\bar{z}) \leftrightarrow \forall \bar{x} X(\bar{x}))$}

here the variables $\bar{z}=z_{1} \ldots z_{n}$ have types $\sigma_{1} \ldots \sigma_{n}$ and $X$ is a variable with the type of a relations between things of types $\sigma_{1} \ldots \sigma_{n} .{ }^{18}$ In order to derive every instance of Logical Necessity from our new principle Quantified Logical Necessity we must make two assumptions. Firstly the schemas

Purity $\operatorname{Pure}^{\sigma}(a)$

Pure Application Pure $\operatorname{Pu}^{\sigma \rightarrow \tau}(F) \wedge \operatorname{Pure}^{\sigma}(a) \rightarrow \operatorname{Pure}^{\tau}(F a)$

where, in the first schema, $a$ is any closed expression that contains only logical vocabulary. And secondly the schemas

\section{Fundamentality}

$$
\begin{aligned}
& \operatorname{Fun}^{\sigma}(c) \\
& c \neq c^{\prime}
\end{aligned}
$$

whenever $c$ and $c^{\prime}$ are distinct non-logical constants of type $\sigma$. The latter two principles correspond to our assumption that we are theorizing in a fundamental language.

Here is one way to think about what Logical Necessity and Quantified Logical Necessity say. We may think of a pure relation $X$ as describing a logical role or pattern — of stating a purely logical relation between its arguments $a_{1} \ldots a_{n}$. The right to left direction of these principles state that if there are some things that occupy a given logical role then it's possible that the fundamental things have that role, and conversely if it's possible that the fundamental things occupy a given logical role that role is in fact occupied.

Let me anticipate one objection to Quantified Logical Necessity, at least in relation to its potential to pin down, or elucidate the notion of a property or relation being fundamental. ${ }^{19}$ For Quantified Logical Necessity is formulated in terms of three notions, logical necessity, purity and fundamentality, and so one might get the sense that we have given a single equation in three unknowns, without enough information to pin the target notions down. But notice that we have already constrained two of the notions in question. For example, our stipulation that $\square$ be the broadest necessity already narrows down the candidates for what could be. Given further assumptions about propositional granularity, one can actually show that the property of being a maximally broad modality is uniquely satisfied (Bacon (2018a)). ${ }^{20}$ Indeed, given these

a given possibility. The model of Quantified Logical Necessity given in the appendix in fact validates the necessitated version of Quantified Logical Necessity, but does so by making the more contentious principle Pure $e^{\sigma \rightarrow t}\left(F_{u n}^{\sigma}\right)$ true.

${ }^{18}$ That is, $\sigma_{1} \rightarrow \sigma_{2} \rightarrow \ldots \rightarrow t$.

${ }^{19}$ Thanks to a referee for pressing me on this issue.

${ }^{20}$ These further assumptions are described in Bacon (2018a), and their consistency with the rest of my theory, are a consequence of the model in the appendix of the present paper. The theory of propositional granularity in question, Adjunctive Booleanism, roughly says that logically equivalent things are the same. It thus ensures Booleanism: the theory consisting of 
assumptions $\square$ can be defined entirely out of the logical operations $\forall_{t \rightarrow t}, \rightarrow$ and $\perp$, so its meaning is entirely pinned down by the logical operations. (As indicated earlier, one such definition of $\square$ is $\lambda p(p=\top$ ), where $=$ may be defined as $\lambda p q . \forall_{t \rightarrow t} X(X p \leftrightarrow X q)$, and $\top$ and $\leftrightarrow$ out of $\rightarrow$ and $\perp$ in the usual way.)

The second primitive of the theory, the notion of a logical, or pure, operation, is one we have a reasonably clear antecedent grasp of. We know, for instance, that the usual logical expressions should denote pure entities — a constraint which we imposed with the schema Purity — and even if the logical operations outstrip those definable from the usual logical operations, we have several theories of what counts as logical that we may appeal to (including the one described in section 5 below). ${ }^{21} \mathrm{~A}$ principal target of our investigation, fundamentality, is thus tightly constrained by its relation to these other notions, as given by Quantified Logical Necessity. ${ }^{22}$

\section{Logical Combinatorialism}

We are now in a position to relate our principle to a metaphysical picture, which I'll call logical combinatorialism. Parts of this picture have already been articulated in a variety of ways by different philosophers, tracing back to Hume's denial of necessary connections. Even though many of these proposals are insufficiently general for my purposes, it will be instructive to survey them.

According to one version of the idea, articulated by Jonathan Schaffer, the fundamental properties and relations are modally free (Schaffer (2010), Wang (2016)). Informally:

Modal Freedom If some fundamental properties and relations can individually be some ways, they can be those ways together.

Another thought in the ball-park is sometimes expressed by the idea that there are no brute necessities. Cian Dorr glosses the idea as follows: "The only genuinely necessary truths [...] are those that reduce, upon

all the higher-order identities stated in $\wedge, \vee$ and $\neg$, where both sides of the identity are equivalent in the propositional calculus (for instance, $\lambda p \lambda q(p \wedge q)=\lambda p \lambda q(q \wedge p)$. And, secondly, Adjunctivism - which states the analogous connection between the quantifiers, $\forall_{\sigma}$ and $\exists_{\sigma}$, and quantificational logic (for instance $\lambda F\left(\forall_{\sigma} x F x\right)=\lambda F\left(\neg \exists_{\sigma} x \neg F x\right)$ ). Adjunctive Booleanism can be precisely described as the result of adding the following identities to higher-order logic:

Adjunctive Booleanism $\lambda \bar{x} A=\lambda \bar{x} B$

when $A$ and $B$ are provably equivalent in the theory $\mathrm{H}$ described in the appendix. Cian Dorr has noted that these equations can in fact be more compactly axiomatized by a smaller set of equations: a finite set of equations of this form for the Boolean identities, and two equations for each quantifier $\forall_{\sigma}$ and $\exists_{\sigma}$. The theory is axiomatized in yet a different way in Bacon (2018a), in terms of the principle of Modalized Functionality: $\square \forall x(F x=G x) \rightarrow F=G$.

${ }^{21}$ But see also Tarski (1986) and Sher (1991).

${ }^{22} \mathrm{~A}$ claim that is further born out when one looks at the model theory, outlined in section 5 , and given more fully in Bacon (forthcoming): only very special elements of a model can be in the extension of Fun ${ }^{\sigma}$ if Quantified Logical Necessity is true. That said, the role of the fundamental is still not always uniquely pinned down - for instance, replacing any fundamental relation with its converse, and leaving the other fundamental entities alone, will result in a distinct 'fundamental basis' — a collection of entities that, if reassigned as the extension of $F u n^{\sigma}$, could still model of Quantified Logical Necessity. The situation is formally quite similar to the role of being a basis for a vector space - only very special sets of vectors may be bases, but a vector space will have several different bases. This analogy is made precise in Bacon (forthcoming). (The situation here is actually a lot better than with vector spaces, for in a vector space any vector is a member of some basis, whereas this is far from the case with fundamental bases.) 
analysis, to truths of logic (in some narrowly-delimited sense of 'logic')" Dorr (2008) p50. This view would be violated, for example, if the property of being an electron and the relation of being duplicates were both fundamental: the necessary truth that duplicates of electrons are electrons would be a brute necessity. On the other hand, if being a duplicate were analysed in terms of sharing certain properties, with electronhood being among them, it would be in some sense a truth of logic that duplicates of electrons are electrons.

We might gloss the idea that there are no brute necessities with the following schema, whose instances are those in which $A$ is a logically consistent sentence:

\section{No Brute Necessities $\diamond A$}

In order for this to be at all plausible we must again make the assumption that the language contains no constants denoting non-fundamental entities, and no codenoting constants. Thus for example, since the sentence $\forall x y(E x \wedge D x y \rightarrow E y)$ ('every duplicate of an electron is an electron') is not a logical truth, $\diamond \neg \forall x y(E x \wedge D x y \rightarrow E y)$ is an instance of our schema. (Of course, we could equally conclude, as Dorr does, that $D$ is not a fundamental predicate and that this sentence is thus not a valid instance of this schema.)

Another principle in the vicinity is the idea that fundamental relations be freely recombinable. Jeff Russell and John Hawthorne propose the following as a partial way to articulate that idea (Russell and Hawthorne (forthcoming)):

Pattern Any actually instantiated pattern is possibly instantiated by the fundamental relations.

Similar ideas are implicit in David Armstrong's combinatorial theory of possibility (Armstrong (1989)), and David Lewis's principle of recombination (Lewis (1986)).

The theme here seems to be that the fundamental properties and relations are extremely plastic with respect to modal space. The notion of modality employed by the above authors is metaphysical modality: so interpreted these principles are quite contentious (see e.g. Wilson (2010), Wang (2016)). My goal here is not vindicate these theses concerning metaphysical modality, and I will remain officially neutral on their status. However, the principles are extremely plausible when reinterpreted in our preferred theory of logical possibility. Indeed, not only are the reinterpreted principles plausible, they are derivable when suitably formalized. ${ }^{23}$

\footnotetext{
${ }^{23}$ The relation between the combinatorial principles interpreted in terms of broad possibility and metaphysical possibility is delicate on account of the fact that there is some disagreement as to which modality occupies the role of metaphysical possibility. Some philosophers have insisted that metaphysical necessity is singled out primarily or solely by its role as 'necessity in the highest degree' (Kripke (1980)) — i.e. the broadest necessity. (This attitude is expressed quite clearly in Fritz (2017): "Yet, it is one of the most central theoretical roles of metaphysical necessity that it is the strongest of the relevant kinds of necessity. Indeed, this may be the most central theoretical role of metaphysical necessity [...] Thus, if there is any such modality as metaphysical necessity, it is the strongest of the relevant kinds of necessity" (pp562-563). See also Williamson (2016).) Such philosophers will often concede that there may be restricted modalities that play some of the other roles that are typically associated with metaphysical necessity, including various essentialist and supervenience theses, but will insist these restricted modalities are highly context sensitive, and unsuited for serious philosophical scrutiny. In any case, if these philosophers are right, there is no distance between theses stated in terms of metaphysical necessity and those stated in terms of broad necessity.
} 
Let's start with a very simple articulation of the combinatorialist idea (see Wittgenstein (1961), Armstrong (1989)). This theory states that whenever you have individuals $a_{1} \ldots a_{n}$ and an $n$-ary relation $R$ there is a possible state of affairs of $a_{1} \ldots a_{n}$ instantiating $R$. Armstrong, for example, is working with a sparse conception of of properties and relations where all properties and relations are assumed to be fundamental. Here is an Armstrong inspired principle:

(1) If $a_{1} \ldots a_{n}$ are fundamental individuals and $R$ a fundamental relation, then possibly $R a_{1} \ldots a_{n}$.

We can derive this from the right-to-left direction of Logical Necessity. The relevant pure relation in this instance is the relation of instantiation, which holds between a relation and some arguments when the arguments instantiate the relation (it can be defined in logical terms as follows: $\lambda X \lambda x_{1} \ldots x_{n} . X x_{1} \ldots x_{n}$ ). Since there exists at least one $n$-ary relation $X$ (e.g. the universal relation), and individuals $x_{1} \ldots x_{n}$ that stand to one another in the instantiation relation it follows by Logical Necessity that it's possible that $a_{1} \ldots a_{n}$ and $R$ stand in the instantiation relation. A straightforward argument shows that logically consistent combinations of atomic facts - propositions stating that fundamental entities stand in fundamental relations - are also possible.

Let's move on to Russell and Hawthorne's version of the principle. Here is an instance of the idea. Consider a love triangle: $a$ loves $b, b$ loves $c$, and $c$ loves $a$ (but not conversely). Since this triangular pattern is instantiated by the unfortunate in love - i.e. there are distinct $x, y$ and $z$ such that $x$ loves $y, y$ loves $z$, and $z$ loves $x$ - it follows that our favourite fundamental relation $R$ (being more massive than, say) possibly instantiates this pattern. It's important to Russell and Hawthorne's conception that we adopt an abundant conception of patterns: they can be highly disjunctive and do not themselves need to be fundamental. We can infer more exotic things like: ${ }^{24}$

(2) It's possible that $R$ has inaccessible order-type.

A relation has inaccessible order-type when its domain is isomorphic to a certain sort of infinite number. Since 'inaccessible well-order' is an instantiated pattern (it is instantiated by some relation - e.g. the relation of being less than on the ordinals), it follows that $R$ possibly instantiates the pattern.

Not every property of a relation counts as a pattern for that relation to instantiate. Russell and Hawthorne restrict to qualitative patterns, meaning, roughly, properties of relations that don't contain essential reference to individuals. But even this is not enough: the property of holding between $x$ and $y$ iff $R$ doesn't hold between $x$ and $y$ for any $x$ and $y$ is a property of relations, and is qualitative if $R$ is, but clearly cannot be instantiated by our fundamental relation $R$. We shall think of a pattern for a relation as a logical or pure property of

\footnotetext{
${ }^{24}$ This example is reminiscent of the sorts of consequences discussed in relation to Lewis's recombination principle.
} 
a relation, where part of our conception of a pure property is one that doesn't make any reference to any fundamental entities at any type (by contrast, we may think of a qualitative property as one that doesn't make reference to fundamental entities of type $e$ ). We precisify this conception of purity in section 5 .

We can derive (2) from Logical Necessity as follows. It turns out that one can define, in (higher-order) logical vocabulary alone, a higher-order predicate Inaccessible $(X)$ which states that a relation $X$ is a wellorder of inaccessible order type (see Shapiro (1991)). ${ }^{25}$ Moreover, it follows from the existence of the standard ordering of the ordinals that $\exists X$ Inaccessible $(X)$. Thus, by the right-to-left direction of Logical Necessity it follows that $\diamond$ Inaccessible $(R)$. This example gives a flavour of the complexity of patterns expressible in higher-order logic alone — i.e. using just $\rightarrow$ and $\forall_{\sigma}$ — without employing a more expansive conception of what is pure.

There is an obvious analogy between Logical Necessity and Russell and Hawthorne's formulations of Pattern. Their idea is that for every set-theoretic model in a signature of fundamental individuals and relations, there is a world where those fundamental individuals and relations instantiate that structure. ${ }^{26}$ Since many mathematical structures can be characterized in higher-order logic, we can similarly prove that the fundamental relations can instantiate those structures. For example, it follows from Logical Necessity that a fundamental predicate $F$ and relation $R$ can have the structure of the natural numbers, where $F$ corresponds to being a number and $R$ to being less than, or the structure of the reals, or even the structure of the set theoretic hierarchy, where $F$ instead corresponds to sethood and $R$ to membership. ${ }^{27}$

On many conceptions of logicality, the properties definable from the logical connectives and quantifiers form only a fragment of all the logical properties. ${ }^{28}$ According to these alternative conceptions, for every mathematical structure for a signature - e.g. a set of ordered pairs for a binary relational predicate — there is a logical property of relations stating that the pattern of instantiation of that relation is of that isomorphism class. Given the hypothesis that the isomorphism class of every set-theoretic model can be characterized by a pure property, we can recover the full strength of Russell and Hawthorne's principle from Quantified Logical Necessity.

But it should be mentioned that our setting is also more general than the Russell-Hawthorne approach, which is phrased in terms of first-order model theory. So far we have only been talking about fundamental relations and properties of individuals. But there might also be fundamental operators — indeed, perhaps

\footnotetext{
${ }^{25}$ That is, Inaccessible $(\lambda x y \cdot x \in y \wedge \operatorname{Ord}(y))$, where Ord the property of being an ordinal and $\in$ is the set membership (which well-orders the ordinals).

${ }^{26}$ Here, and throughout, a signature refers to a specification of the non-logical constants of each type. A first-order signature thus tells us what the individual constants, and predicates of each arity are.

${ }^{27}$ The last point follows if the size of the universe is expressible in higher-order logic, since second order ZFC with any claim maintaining that the universe has a certain size is a categorical theory, and can be expressed by a single sentence of higher-order logic in a relation and predicate variable, for membership and sethood respectively.

${ }^{28}$ Including the definition we offer in section 5. See also Tarski (1986), Sher (1991).
} 
metaphysical necessity is such an operator. While Russell and Hawthorne's principle entails that being more massive than is freely recombinable, with the force of metaphysical modality, it certainly doesn't entail that metaphysical necessity is freely recombinable in same sense.

By contrast, given the assumption that metaphysical modality is fundamental, Logical Necessity says, putting it loosely, that any model of metaphysical modal reality could (in the sense of logical possibility) be how metaphysical modal reality is. For example, the property of being non-truthful, $\lambda X \exists p(X p \wedge \neg p)$ is logical, and moreover there are non-truthful operators (like negation). Thus it follows by Logical Necessity that:

(3) It's logically possible that metaphysical necessity is not truthful.

Indeed, this gives us an argument that logical necessity is not metaphysical necessity, given the assumption that metaphysical necessity is fundamental, for it is clearly not metaphysically possible that metaphysical necessity is non-truthful. ${ }^{29}$ Similarly, our framework allows for patterns involving fundamental quantifiers, predicate modifiers, and so on.

There are various strengthenings of the simple principle Pattern that are also delivered by our principle. For example, so far we have only appealed to instances of Logical Necessity involving one fundamental constant. But we can also establish the possibility of patterns that state connections between the fundamental. For example, Logical Necessity implies that two fundamental relations, $R$ and $S$, could have been converses of one another: this is because being the converse of is a logical relation $(X=\lambda x z . Y z x)$ which is instantiated by any relation and its converse, and so $R$ and $S$ are possible converses given the right-to-left direction of Logical Necessity. Of particular interest is a limiting case of a pure relation: identity. If two fundamental entities belong to the same type then Quantified Logical Necessity dictates that they must be possibly identical, for the relation of identity is both logical, and instantiated (since something is identical to something). Thus we may derive the schema: ${ }^{30}$

(4) $\operatorname{Fun}^{\sigma}(x) \wedge \operatorname{Fun}^{\sigma}(y) \rightarrow \diamond x=y$

It is worth pausing for a minute to examine (3), since it has consequences that are unfamiliar to many contemporary metaphysicians. For one thing, it implies that distinctness can be a logically contingent matter. For instance, it entails that any two distinct fundamental individuals, $a$ and $b$, are possibly identical. It thus also implies that the Brouwerian axiom, which states that whatever is true is necessarily possible, has counterinstances. For, as Prior (1962) (pp.206-207) observed, given the Brouwerian axiom one can prove

\footnotetext{
${ }^{29}$ Alternatively, one might take this argument to demonstrate that metaphysical necessity is not fundamental, just as we must conclude that the notion of logical necessity is not fundamental. Earlier we mentioned that give certain assumptions the logical necessity (in virtue of being the broadest necessity) is definable in terms of logical vocabulary (as, e.g., $\lambda p . p=\top$ ) - a reason to think that logical necessity is in fact pure, not fundamental.

${ }^{30}$ Here $x$ and $y$ are variables of type $\sigma$, and $=$ the relation of identity between type $\sigma$ things.
} 
the necessity of distinctness. ${ }^{31}$ Thus the logic of Logical Necessity is not the familiar logic of S5, often thought to be the logic of metaphysical necessity. ${ }^{32}$ (This is not to say, however, that that our logic needs to be non-classical: the contingency of distinctness is consistent with classical higher-order logic, including Leibniz's law and the necessity of identity.)

It's important to emphasize that while these consequences reveal that we are dealing with an unfamiliar modality, they do not reveal it to be an ill-conceived or incoherent one. ${ }^{33}$ Firstly, nothing compels us to identify the notion of logical necessity we have introduced here with metaphysical necessity. (3) is entirely consistent with the metaphysical necessity of distinctness, and with the usual principles of S5 as a logic of metaphysical necessity. Secondly, it's worth reiterating that we have introduced this modality by a particular logical role, Logical Necessity. It was not a concept we necessarily had antecedently, and any pretheoretic judgments we have about how it should behave ought to be taken with that in mind. My attitude is that it is better to set these untutored intuitions aside and follow the theory where it leads, judging the result by its fruits, familiarizing oneself with the unfamiliar if necessary. Finally, our motivating remarks do go some way to making this result unsurprising, for this logical modality is supposed to stand to reality as logical truth stands to language: but clearly logic has nothing to say about whether two objects, $a$ and $b$, are identical or not, resembling the fact that no identity statement involving distinct names is a logical truth. Indeed, recall that we have defined $\square A$ as $A=\top$, for a tautology $\top$. So read, the necessity of distinctness implies that the the proposition that Mars is distinct from Venus is the same proposition as a tautology. Such propositional identities are hardly obvious, and competing hyperintensional theories of content count far less as tautologous (for instance Fine (2017), or Dorr (2016)). ${ }^{34}$

So far we have only used the right-to-left direction of Logical Necessity. Consider Modal Freedom as

\footnotetext{
${ }^{31}$ The Brouwerian axiom, $\mathrm{B}$, states: $A \rightarrow \square \diamond A$. Indeed, one can prove the necessity of distinctness from $\mathrm{B}^{n}$ or $\mathrm{B}^{<\omega}$

$\mathrm{B}^{n} \quad A \rightarrow \square \diamond^{n} A$

$\mathrm{B}^{<\omega} \quad \square(A \rightarrow \square A) \rightarrow(\diamond A \rightarrow A)$

where $\diamond^{n}$ represents a sequence of $n \diamond_{\mathrm{S}}$ in a row (and nothing when $n=0$ ). $\mathrm{B}^{n}$ is validated by frames such that, whenever $x$ sees $y$, one can get back from $y$ to $x$ in $n$ hops of the accessibility relation (thus $\mathrm{B}=\mathrm{B}^{1}$ is validated in symmetric frames), and, in reflexive frames, $\mathrm{B}^{<\omega}$ corresponds to the condition that one can get back in any finite number of hops. Thus these weakenings of $\mathrm{B}$ must all be reliquished as well.

${ }^{32}$ One might alternatively take the necessity of distinctness, or the Brouwerian axiom as a postulate, and in conjunction with Logical Necessity prove that there can be at most one fundamental entity in any give type. Our official theory is technically neutral about the contingency of distinctness, but the existence of multiple fundamental entities in a given type - especially in the type of individuals - strikes me as a reasonable enough working assumption to warrant setting the necessity of distinctness and the Brouwerian axiom aside.

${ }^{33}$ Some allegations of incoherence in relation to contingent distinctness and failures of the Brouwerian axiom are addressed in Bacon (2018a).

${ }^{34} \mathrm{~A}$ referee has suggested that we might avoid the failures of $\mathrm{B}$ and the contingency of distinctness by restricting the quantifiers in $\forall x_{1} \ldots x_{n} A\left(x_{1} \ldots x_{n}\right)$, in the right-hand-side of Quantified Logical Necessity, so that $x_{1} \ldots x_{n}$ are all distinct (in accordance with the considerations in Fine (1989)). Although one could derive many of the recombinatorialist ideas with this variant, the structural ideas, derived in the next section, would not follow, robbing the theory of one of its attractive elements. Note also that B is directly undermined by our analogy between $\square$ and logical truth, independently of the contingency of distinctness. For, letting $F$ be a non-logical predicate, we see that neither $\diamond \exists x F x$ or $\diamond \neg \exists x F x$ is a logical truth (the former, by reinterpreting $F$ with the inconsistent property, $\lambda x \perp$, and the latter by the tautologous property, $\lambda x \top$ ). But the Brouwerian axiom allows one to derive $(\square \diamond \exists x F x) \vee(\square \diamond \neg \exists x F x)$ from an instance of excluded middle for $\exists x F x$, and reasoning by cases.
} 
formulated in the current context, where $R$ and $S$ are again assumed to be distinct and fundamental, and $P$ and $Q$ are patterns (i.e. are pure properties of relations):

(5) If it's possible that $R$ has pattern $P$ and possible that $S$ has pattern $Q$ then it's possible that $R$ has $P$ and $S$ has $Q$.

Here we need the left-to-right direction of Logical Necessity, which allows us to get from the antecedent of (5) to $\exists X P(X)$ and $\exists Y Q(Y)$. Thus $\exists X Y(P(X) \wedge Q(Y)$ and so $\diamond(P(R) \wedge Q(S))$ by the right-to-left direction of Logical Necessity.

Finally we can prove that every instance of No Brute Necessities is true from the truth of Logical Necessity. According to our definition of sentential logical consistency, $A(\bar{c})$ is logically consistent iff $\exists \bar{x} A(\bar{x})$ is true. Assuming that Logical Necessity is true, it follows that $\diamond A(\bar{c})$ is true.

We have illustrated our examples with fundamental relations and unary patterns, but this is not essential. A generalization of (5) states that if $c_{1} \ldots c_{n}$ and $d_{1} \ldots d_{m}$ are fundamental and distinct entities of any type, and possibly $c_{1} \ldots c_{n}$ stand to one another in the pattern $P$, and $d_{1} \ldots d_{m}$ in the patter $Q$, then they possibly stand in that pattern together: $\diamond P\left(c_{1} \ldots c_{n}\right) \wedge \diamond Q\left(d_{1} \ldots d_{m}\right) \rightarrow \diamond\left(P\left(c_{1} \ldots c_{n}\right) \wedge Q\left(d_{1} \ldots d_{m}\right)\right)$. This, and generalizations of the other examples we have discussed, are proven in exactly the same manner.

Thus we can see that Logical Necessity brings together a number of combinatorialist ideas about the fundamental properties and relations. It ensures that modal reality is sufficiently rich, and ensures that the fundamental entities are sufficiently independent of one another. It is simple to state, and strong. Moreover, it is parsimonious: it is stated in logical terms, and doesn't tie the structure of modal reality to the structure of sets. By contrast Lewis's principle of recombination, and the Russell-Hawthorne Pattern principle, rely on set theory to capture the sense in which modal reality is as big as possible. After all, why should questions about the size of modal reality be hostage to the answers to questions about the size of mathematical reality?

\section{Fundamentality and Structure}

In this section we shall implement our theory of logical possibility in order to get some purchase on the notion of fundamentality. Recall again the metaphor of reality as a language, and the fundamental as the primitives of that language. We have explored one sense in which the fundamental might be like the primitive constants: the fundamental are freely recombinable with respect to logical possibility by analogy with the way that the interpretations of primitive constants are unconstrained in model theory.

But the metaphor conjures up a more specific picture than the combinatorialist idea apparently delivers alone. The picture is a structural one, in which propositions, properties and relations are built out of the 
fundamental properties and relations via logical operations, in the same way that arbitrary sentences and predicates may be built out of the primitive non-logical constants via the logical operations and rules of syntax. Could the combinatorialist theory of possibility outlined in the last three sections shed light on this structural conception of reality?

In his paper 'To be $F$ is to be $G$ ', Cian Dorr considers the possibility that reality is structured as a language is. ${ }^{35}$ He examines a class of principles, formulated in higher-order logic, which he takes to capture the structural vision of reality. Consider, for instance, the following principle:

Structure $F a=G a \rightarrow F=G$

where $F$ and $G$ have type $\sigma \rightarrow \tau$, and $a$ is any term of type $\sigma$. By instantiating $F, G$ and $a$ appropriately, this principle allows us to infer from the assumption that the proposition that John is a lawyer is the same as the proposition that John is an attorney, that being a lawyer and being an attorney are the same property. For, intuitively, the proposition that John is a lawyer has exactly two constituents - John, and the property of being a lawyer - and so any proposition identical to it must have the same constituents.

Dorr then goes on to consider a number of objections to the structural vision of reality, the most damning being that the principle Structure is inconsistent in an extremely minimal system of higher-order logic. ${ }^{36} \mathrm{I}$ take this to be a decisive reason to reject the principle Structure. ${ }^{37}$ But this takeaway is consistent with many broadly structural insights about the nature of reality. And, more importantly in my view, it's possible that many of the insights that fall out of the structural picture can be upheld in a theory of propositional granularity that isn't overtly structural, and may be used to helpfully elucidate the notion of fundamentality.

According to the structured picture there are both structured and unstructured entities. The unstructured entities are, like the non-logical constants of a language, simple in the sense that they do not contain any proper constituents. If the structured account were true, these would be prime candidates for being the fundamental entities. There are also structured entities that contain these fundamental entities as constituents. Just like expressions in a language, given any proposition, property, or what have you, you may ask what its fundamental constituents are, and how it is logically built out of them. Thus we have roughly two characteristic structural ideas:

1. Any proposition, property, et cetera, can be decomposed uniquely into fundamental constituents via

${ }^{36}$ Dorr does not consider Structure directly, but a related principle that he calls Propositional Structure. Dorr's argument against Propositional Structure applies, without modification, to an instance of Structure where $F$ and $G$ are operators, and $a$ a sentence.

${ }^{37}$ There are maneuvers one can make to preserve Structure, which I shan't take up here. These involve either weakening classical propositional logic along the lines suggested for other related paradoxes, like the liar and Russell's paradox (Kripke (1975), Priest (2006), Field (2008)), or weakening the logic of quantification (as Russell himself does Russell (1908), and more recently Tucker and Thomason (2011)). For further discussion of the latter option, especially as it relates to ramification, see Hodes (2015) and Bacon et al. (2016).
} 
logical operations.

2. The fundamental are simple and cannot be defined non-trivially out of other fundamental constituents.

While the above are all delivered by the structural vision, we will now argue that versions of these ideas all fall out of the present theory of logical possibility. This is somewhat unexpected, since the combinatorialist ideas defended above are not usually related to these structural principles about propositions and properties.

The foregoing remarks trade on this notion of decomposing, or defining one set of entities out of another. We already have a fairly precise model of what this means for the case of expressions in a language, and for our purposes, expressions will be a good enough proxy for structured properties and relations. Suppose I have some closed expression, $M$, of higher-order logic that contains the non-logical constants $c_{1} \ldots c_{n}$ : in this case we say that $M$ is defined from $c_{1} \ldots c_{n}$, via the logical operations. It is also helpful to have a convenient way to talk about the way in which $M$ is defined, via the logical operations, from $c_{1} \ldots c_{n}$. Intuitively, we should think of the way in which $M$ is defined from $c_{1} \ldots c_{n}$ as the result of 'punching holes' in $M$ where the constants appear, leaving behind a perforated logical expression that yields $M$ when $c_{1} \ldots c_{n}$ are appropriately reinserted. Luckily there is a trick available in higher-order logic for making this precise: one can re-express any term $M$ in non-logical constants $c_{1} \ldots c_{n}$ as a logical operation applied to its non-logical constituents. The trick is this: take each constant $c_{i}$ in $M$ and replace it with a variable of the same type, $x_{i}$, and prefix the result with a string of $\lambda \mathrm{s}: \lambda x_{1} \ldots \lambda x_{n} \cdot M\left[x_{1} / c_{1} \ldots x_{n} / c_{n}\right]$, or using our earlier conventions $\lambda \bar{x} \cdot M[\bar{x} / \bar{c}]$. We shall abbreviate the result of performing this operation to $M, \lambda M$. The result is a logical expression: a closed expression that only contains bound variables and logical operations. Moreover, it is an expression that takes $n$ arguments, matching the types of $c_{1} \ldots c_{n}$, to produce something with the same type as $M$. Higher-order logic - more specifically, the principle $\beta$-equivalence discussed below - allows us to prove the identity $(\lambda M) c_{1} \ldots c_{n}=M$. Thus we may think of $M$ as being defined out of the constants $c_{1} \ldots c_{n}$ via the logical operation $\lambda M$.

In order to make sense of the idea that a property or relation is built up of, or 'defined' out of some fundamental properties and relations, it is natural to look for a notion that stands to reality as the notion of definability stands to language. That is, we would like a notion of metaphysical definability. ${ }^{38}$ Wielding the above trick, we shall say that $y$ is metaphysically definable from $x_{1} \ldots x_{n}$ if and only if there is some logical operation (the analogue of $\lambda M$ above) that yields $y$ when applied to $x_{1} \ldots x_{n}:{ }^{39}$

\section{Metaphysical Definability $M D(y, \bar{x}):=\exists X(\operatorname{Pure}(X) \wedge y=X \bar{x})$}

\footnotetext{
${ }^{38}$ I am repurposing this terminology slightly from Bacon (2019), which uses the term for a special case of the notion developed here and in Bacon (forthcoming).

${ }^{39}$ In the below $x_{1} \ldots x_{n}$ have types $\sigma_{1} \ldots \sigma_{n}, y$ has type $\tau$ and $X$ type $\sigma_{1} \rightarrow \ldots \rightarrow \sigma_{n} \rightarrow \tau$. Observe, then, that metaphysical definability is really a class of relations for each choice of types $\sigma_{1} \ldots \sigma_{n}, \tau$.
} 
If $A$ is a closed expression defined from the non-logical constants $\bar{c}=c_{1} \ldots c_{n}$, then we may prove, as desired, that $M D(A, \bar{c})$ - the definability witnessed by the logical operation $\lambda A$ (ensured to be pure by our earlier assumption, Purity, that closed logical expressions denote pure things).

It should be noted that we have been discussing the special case of metaphysical definability where $x_{1} \ldots x_{n}$ are fundamental. But our definition is not restricted to this case: one can ask about the relative definability of non-fundamental things in terms of others, as one might if one was investigating which properties and relations studied by the special sciences are more fundamental than which.

While we motivated our definition by contemplating the structural/linguistic model of reality, note that our definition uses only concepts we have already introduced in stating our preferred version of combinatorialism. Namely, the notion of a pure operation. And, as we saw in section 3, we already have a powerful theory governing the notion of purity, namely Quantified Logical Necessity; thus we already have a powerful theory of metaphysical definability, even if we didn’t originally conceive of it as such. With the notion of metaphysical definability in hand we may examine the two features of the structural vision we mentioned above. The first is that every proposition, property, relation, et cetera, may be decomposed uniquely into fundamental constituents by logical operations. There are two components of this idea corresponding to the existence and the uniqueness of such a decomposition.

Let's begin with uniqueness. Suppose that I have some proposition, which is metaphysically definable from some fundamental entities $z_{1} \ldots z_{n}$ via a logical operation $X$ : thus our proposition may be written $X \bar{z}$. Part of the uniqueness idea is that there can't be any other way to logically combine fundamental entities $z_{1} \ldots z_{n}$ to get this proposition. That is, if $Y$ is another way of combining these fundamental entities, $Y \bar{z}$ also yields the original proposition, $X \bar{z}$, then $Y=X$, where $X$ is the original way of combining $z_{1} \ldots z_{n}$. That is we should have the following principle: ${ }^{40}$

\section{Quantified Separated Structure $\forall X Y \forall \bar{z}(\operatorname{Pure}(X) \wedge \operatorname{Pure}(Y) \wedge F u n(\bar{z}) \rightarrow(X \bar{z}=Y \bar{z} \rightarrow X=Y)$}

Recalling that $\operatorname{Fun}(\bar{z})$ is short for the conjunction stating that $z_{1} \ldots z_{n}$ are fundamental and pairwise distinct.

Strikingly Quantified Separate Structure is a direct consequence of our combinatorial principle Quantified Logical Necessity given some background higher-order logic. For suppose $X$ and $Y$ are pure, $\bar{z}$ fundamental and distinct, and $X \bar{z}=Y \bar{z}$. Since identities can be proven to be necessary (given Leibniz's law), we get that $X \bar{z}=Y \bar{z}$ implies $\square(X \bar{z}=Y \bar{z})$ which entails by Quantified Logical Necessity that $\forall \bar{z}(X \bar{z}=Y \bar{z})$. Since this sentence contains no constants, we may apply Quantified Logical Necessity to get $\square \forall \bar{z}(X \bar{z}=Y \bar{z})$. Modalized Functionality is the principle that if functional entities, $X$ and $Y$, necessarily output identical

\footnotetext{
${ }^{40}$ Quantified Separated Structure is type schematic, and has an instance for each choice of types for the variables that yields a (well-typed) sentence.
} 
things for every input, they are the same (see Bacon (2018a)). It allows us to infer that $X=Y$, as required. ${ }^{41}$ Like Quantified Logical Necessity, Quantified Separated Structure has a schematic variant, that follows from it immediately given the assumptions Purity and Fundamentality from section 2:

(*) $F a_{1} \ldots a_{n}=G a_{1} \ldots a_{n} \rightarrow F=G$ provided $F$ and $G$ are closed and contain only logical vocabulary, and $a_{1} \ldots a_{n}$ are distinct fundamental constants.

which is easily seen to be a equivalent to a special case of Structure: ${ }^{42}$

Separated Structure $F c=G c \rightarrow F=G$ provided $c$ is a fundamental constant that doesn't appear in $F$ or $G$.

(Here, as with Logical Necessity, we assume that we are theorizing in a fundamental language, and $F$ and $G$ are closed.) The restricted version of structure avoid some superficial counterexamples to Structure, also discussed by Hodes (2015) and Dorr (2016), that arise when one assumes a principle that is standardly assumed to govern $\lambda$-expressions called $\beta$-equivalence. $\beta$-equivalence states that the result of applying a $\lambda$ expression $\lambda x . M$ to an argument $a$ should be the same as substituting $a$ for $x$ in $M: M[a / x]{ }^{43}$ For example, supposing the property of being ancient and wise is formalized with a $\lambda$-expression, $\lambda x(x$ is ancient $\wedge$ $x$ is wise), then our principle guarantees that the proposition that Socrates is ancient and wise and the proposition that Socrates is ancient and Socrates is wise (formalized $a$ is ancient $\wedge a$ is wise) are the same. Given this principle it follows that a proposition such as Mary loves Mary, may be decomposed in multiple ways: for example, it can be equivalently thought of as the property of loving Mary $(\lambda x$ ( $x$ loves Mary)) applied to Mary, as the property of being loved by Mary $(\lambda x$ (Mary loves $x)$ ) applied to Mary, and the property of loving oneself $(\lambda x(x$ loves $x))$ applied to Mary. ${ }^{44}$ It can even be thought of as the vacuous property of being such that Mary loves Mary ( $\lambda x$ (Mary loves Mary)) applied to Mary (or applied anyone else, for that matter). These are all different decompositions because the property of being loved by Mary, loving Mary, loving oneself, and the vacuous property are all distinct (they even have different extensions). Note, however, that there is only one decomposition of the proposition that Mary loves Mary into a unary property that doesn't involve Mary applied to Mary, and so the above remarks are all consistent with

\footnotetext{
${ }^{41}$ Modalized Functionality, and it's unmodalized variant, $\forall z(X z=Y z) \rightarrow X=Y$, should not be confused with the principle of extensionality, which says that extensionally equivalent properties and relations are identical.

${ }^{42}$ Recall that Purity stated that closed purely logical expressions denote pure entities, and Fundamentality that the constants denote distinct fundamental entities, so we have the assumptions Pure $(F)$, Pure $(G)$ and $F u n(\bar{a})$ needed to infer $(*)$ immediately from Quantified Separated Structure. Separated Structure follows from (*) by noting that $F c$ and $G c$ can be decomposed into logical expressions $(\lambda F)$ and $(\lambda G)$ - obtained by $\lambda$ ing out the constants in $F$ and $G$ - applied to the sequence of fundamental constants, $\bar{a} c$, where $\bar{a}$ enumerate the constants appearing in $F$ and $G$. (So that both expressions may be applied to the same sequence of fundamental constants, we may have to conceive of $\lambda F$ as having vacuous $\lambda$ abstracts if a constant appears in $G$ that doesn't appear in $F$, and similarly for $\lambda G$.)

${ }^{43}$ Provided $a$ doesn't contain free variables that get bound when this substitution is made; this sort of thing can always be avoided by relabeling bound variables.

${ }^{44}$ See also the discussion of $\beta$-equivalence in Hodes (2015) and Dorr (2016).
} 
Separated Structure, which states the uniqueness of a decomposition of the proposition that Mary loves Mary into Mary and a Mary-free property. The consistency of the entire package, including Separated Structure and $\beta$-equivalence, is a consequence of the model construction in the appendix. ${ }^{45}$

The above highlights a more general obstacle to a stronger uniqueness thought, however. For $\beta$ equivalence allows us to create logical operations that reorder, duplicate, and throw away arguments. Every binary relation $R$, has a converse, $C R$ (defined as $\lambda x \lambda y . R y x$ ). If $R$ is pure, then so is $C R$, thus if, for example, a proposition $p$ may be decomposed as applying $R$ to two fundamental arguments, $a$ and $b, R a b$, it may also be decomposed by applying the converse of $R$ to $b$ and $a,(C R) b a$. Similarly, every unary property, $F$, gives rise to a binary relation $K F$ (defined $\lambda y \cdot F$ ) that forgets one of its arguments, and which is also pure if $F$ is. So if a proposition can be decomposed into a pure property $F$ applied to a fundamental argument $a, F a$, then it can also be decomposed into a pure relation that throws its first argument away, with an extra (redundant) argument $(K F) b a$. Finally, every binary relation $R$ gives rise to a unary property, the reflexivization of $R, W R$ (defined $\lambda y$.Ryy), that duplicates its only argument and feeds to $X$ twice, which, for instance, takes the binary relation of loving to the unary property of loving oneself. So anything that can be decomposed by applying a pure operation to the same argument twice, Raa, can also be decomposed by applying that operations reflexization to that argument once, $(W R) a$.

One option is to simply give $\beta$-equivalence up, as suggested in Dorr (2016) in response to similar issues. Yet the principle is powerful and predictive, and one might argue, a compulsory part of our adopting the $\lambda$ notation in the first place. ${ }^{46}$ I will not attempt to launch a proper defense of $\beta$-equivalence here, or to argue against approaches that reject it: but I will adopt $\beta$-equivalence as a working assumption, if only to delimit the scope of this investigation. With that in mind, we will explore a weakening of the uniqueness idea that is consistent with $\beta$-equivalence: namely that a proposition (property, operator, et cetera) may be decomposed into its fundamental constituents uniquely modulo operations that re-order, duplicate, or throw away arguments. One way to do this is to ask whether an entity $y$ can be decomposed uniquely into a logical operation $X$ relative to a sequence $z_{1} \ldots z_{n}$ of fundamental entities, where this list does not contain duplicates, and, because it is a sequence, is given in a particular order and has a particular length. On this conception, some of the fundamental elements $z_{1} \ldots z_{n}$ may not be essential to the decomposition, in the sense that the entity might also be decomposable into a logical operation applied to some shorter sequence with that fundamental element omitted. This happens exactly in the case where the logical part of the

\footnotetext{
${ }^{45}$ There are some suggestive remarks in favour of this version of the unique decomposition thought in Fitch (1952) Chapter 4, §17. See Humberstone (2000) for further discussion of Fitch's remarks.

${ }^{46}$ It should be noted that Dorr (2016) takes issue only with $\beta$-equivalence regarding its verdicts in the second case, involving thrown away arguments. He maintains a restricted version of $\beta$-equivalence that allows one to reorder and duplicate arguments. (In fact, Dorr restricts his language so one can't even form vacuous $\lambda$-abstractions, so that one might continue taking $\beta$ equivalence to be constitutive of using this version of the $\lambda$-notation.)
} 
decomposition throws away some of its arguments, so we see that fixing the length of the decomposition controls for forgetful operations. Similarly, the fact that we ask for a decomposition relative to a particular order of the arguments controls for the multiplicity of decompositions due to re-ordering, and the fact that we have ruled out duplicates from the sequence controls for multiplicity due to argument duplication. The resulting quasi-uniqueness principle thus states that if $X$ and $Y$ are pure, and $z_{1} \ldots z_{n}$ are fundamental and distinct, and $X z_{1} \ldots z_{n}=Y z_{1} \ldots z_{n}$ then $X=Y$. This is, of course, exactly what Quantified Separated Structure says.

One might also ask for a more fine-grained analysis. Say a pure property $X$ is minimal if it isn't forgetful in any of its arguments. ${ }^{47}$ A more fine-grained principle states that if $X$ and $Y$ are pure and minimal, $z_{1} \ldots z_{n}$ are fundamental and distinct, $w_{1} \ldots w_{n}$ are all fundamental and distinct, and $X z_{1} . . z_{n}=Y w_{1} \ldots w_{n}$ then $X$ and $Y$ are (generalized) converses of one another, and the arguments $z_{1} \ldots z_{n}$ are the same as $w_{1} \ldots w_{n}$ modulo the order in which $X$ and $Y$ are converses. ${ }^{48}$ This principle may be formalized (as in the last two footnotes) and proven in the present system, although I omit the details for the sake of brevity. The schematic version of Separated Structure in a fundamental language has an even simpler generalization, which neatly illustrates the cases we just discussed. Let $a_{1} \ldots a_{n}, b_{1} \ldots b_{m}$ be a sequence of constants, possibly with repetitions, and let $\pi$ be a bijective association of variables $\left\{x_{1} \ldots x_{k}\right\}$ to fundamental constants in $\left\{a_{1} \ldots a_{n}, b_{1} \ldots b_{m}\right\}:$

General Separated Structure $F a_{1} \ldots a_{n}=G b_{1} \ldots b_{m} \rightarrow \lambda x_{1} \ldots x_{k} . F\left(\pi a_{1}\right) \ldots\left(\pi a_{n}\right)=\lambda x_{1} \ldots x_{k} . G\left(\pi b_{1}\right) \ldots\left(\pi b_{m}\right)$ provided none of $a_{1} \ldots a_{n}, b_{1} \ldots b_{m}$ appears in $F$ or $G$. It is another consequence of Logical Necessity, this time noting that, in the special case where $F$ and $G$ are logical, $F \bar{a}=G \bar{b} \leftrightarrow \forall \bar{x} . F \pi \bar{a}=G \pi \bar{b}$ is an instance of Logical Necessity (the more general case being treated as before). Among its instances are the following, where $a$ and $b$ are fundamental constants, and $R, S$ and $F$ are closed terms not containing $a$ or $b$ :

$$
\begin{aligned}
& R a b=S b a \rightarrow \lambda x y \cdot R x y=\lambda x y \cdot S y x \\
& R a b=F b \rightarrow \lambda x y \cdot R x y=\lambda x y \cdot F y \\
& R a a=F a \rightarrow \lambda x \cdot R x x=\lambda x . F x
\end{aligned}
$$

\footnotetext{
${ }^{47}$ This may be made precise as follows. Suppose that $X$ has type $\sigma_{1} \rightarrow \ldots \sigma_{n} \rightarrow \tau$ where $\tau$ is $e$ or $t$. Let us write $K^{i} X$ for the expansion of $X$ with a redundant argument in the $i$ th position: $K^{i} X:=\lambda x_{1} \ldots x_{n} . X x_{1} \ldots x_{i-1} x_{i+1} \ldots x_{n}$. We can capture the idea that $X$ is minimal with the finite conjunction:

$$
\bigwedge_{i} \neg \exists Y_{i}\left(X=K^{i} Y_{i}\right)
$$

where $Y_{i}$ is a variable of type $\sigma_{1} \rightarrow \ldots \sigma_{i-1} \rightarrow \sigma_{i+1} \rightarrow \ldots \tau$.

${ }^{48}$ Given a permutation $\pi$ of $\{1 \ldots n\}$, the $\pi$ converse of $X$ written $C^{\pi} X$ is defined as $\lambda x_{1} \ldots x_{n} . X x_{\pi 1} \ldots x_{\pi n}$. Thus the claim that $X$ and $Y$ are generalized converses, and that $z_{1} \ldots z_{n}$ is the corresponding reordering of $w_{1} \ldots w_{n}$ is formalized as:

$$
\left(\bigvee_{\pi}\left(X=C^{\pi} Y \wedge \bigwedge_{i} z_{i}=w_{\pi i}\right)\right)
$$
}


Thus, for instance, the first instances tells us that the only way $R a b$ and $S b a$ can be the same is if $R$ and $S$ are converses of each other. (Simplifying using the $\lambda$-calculus a little, this is equivalent in our earlier notation to saying $R=C S$. With those same conventions, the second tells us that if $R a b=F b$ then $R=K F$, and the third that if $R a a=F a$ then $W R=F$.)

Let's turn to the existence of fundamental decompositions. Unlike the uniqueness component, the existence of a decomposition for every entity into fundamental entities by a logical operation is not guaranteed by our theory of logical necessity alone: we need to supplement it. Here is the informal idea: for any $y$ of type $\tau$ we want to be able to find fundamental entities $z_{1} \ldots z_{n}$ in some types $\sigma_{1} \ldots \sigma_{n}$ such that $y$ is metaphysically definable from $z_{1} \ldots z_{n}$ (i.e. $y$ is constructed from $z_{1} \ldots z_{n}$ via a logical operation). This sort of informal idea, however, cannot be formalized in higher-order logic since it involves the sort of quantification over types that higher-order logic forbids. However stronger assumptions with the same sort of force can be formulated in higher-order logic: given a finite list of types, $\sigma_{1} \ldots \sigma_{n}$, one can state in higher-order logic the claim that every proposition can be decomposed into fundamental entities of types $\sigma_{1} \ldots \sigma_{n}$ and a logical operation. Moreover, such a principle is plausible if the number of fundamental properties and relations is finite and belong to types $\sigma_{1} \ldots \sigma_{n}$ when suitably enumerated. For a given choice of types for the fundamental, let us call this principle Fundamental Completeness: ${ }^{49}$

\section{Fundamental Completeness $\forall x \exists Y \exists \bar{z}(\operatorname{Pure}(Y) \wedge F u n(\bar{z}) \wedge x=Y \bar{z})$}

Although Fundamental Completeness is a natural principle that is worth exploring further, it is not officially part of the package of views being investigated here. Firstly, it is worth noting that its consistency with the rest of theory is unknown, and it is not true in the model sketched in the appendix. If Logical Necessity and Fundamental Completeness turned out to be inconsistent, it is my view that Fundamental Completeness should be the one to go. Furthermore, there are philosophical views that rule it out. For example, one might think that a complete list of fundamental entities will not be confined to any finite collection of types. But even supposing that they are, there are views about moral facts and vagueness that conflict with Fundamental Completeness. For example, according to Schiffer (2010) and Bacon (2018b), there are vague propositions. It's plausible, however, that both logical and fundamental entities are precise. Fundamental Completeness would then entail that all propositions are the result of applying a precise logical operation to precise fundamental entities. Since precision is presumably closed under application, this would imply that all propositions are precise. ${ }^{50}$ Fundamental Completeness should also be rejected if you thought that

\footnotetext{
${ }^{49}$ Here $\bar{z}=z_{1} \ldots z_{n}$ is a sequence of variables of type $\sigma_{1} \ldots \sigma_{n}, Y$ a variable of type $\sigma_{1} \rightarrow \ldots \rightarrow \sigma_{n} \rightarrow \tau$ and $x$ a variable of type $\tau$. Note that if $p$ can be constructed from a proper subset of the fundamental entities via a logical operation $X$, then it can also be constructed from all of the fundamental entities by a logical operation $\lambda x_{1} \ldots \lambda x_{k} X$ that ignores its first $k$ arguments.

${ }^{50}$ One might draw a different moral from this. In Bacon (2018b) I argue that one cannot both maintain (i) that the fundamental properties and relations are precise and (ii) that it is a precise matter which the fundamental properties and
} 
moral propositions cannot be defined from purely logical and physical properties, and that all fundamental properties are physical; one might think of this as a precisification of the idea that you can't get an ought from an is.

The other structural idea that we listed is the idea that the fundamental properties are simple, in the same sense that the constants of a language are simple. Constants are simple because they cannot be defined out of one another, in contrast with the complex, defined expressions. Thus we might gloss metaphysical simplicity by the idea that fundamental properties and relations cannot be metaphysically defined out of one another.

It's worth emphasizing that the idea that the fundamental properties cannot be defined in simpler terms is a widespread assumption accepted even by those who do not adopt an overtly structural account of propositions and properties. David Lewis, for example, writes of the perfectly natural properties that 'there are only just enough of them to characterise things completely and without redundancy' Lewis (1986), p60. The no-redundancy constraint here just means that it's not possible to define one fundamental relation out of the others. To illustrate, suppose that being an electron, being a proton, being the fusion of and being bound by are all fundamental properties or relations, and that being a hydrogen atom is just being the fusion of an electron and a proton that are bound by the Coulomb force. ${ }^{51}$ Then the no-redundancy thought says that being a hydrogen atom is not fundamental, since it can be defined out of other fundamental properties and relations. ${ }^{52}$ Lewis is presumably appealing to a more coarse-grained notion of definability than a structured proposition theorist would. I will now show that this idea is closely related to Hume's dictum (endorsed by Lewis) that there are no necessary connections between fundamental properties and relations; it is thus natural to want the independence of the fundamental to fall out of the present theory.

The independence of the fundamental says that if $y$ is fundamental, and $x_{1} \ldots x_{n}$ are distinct from $y$ and fundamental, then $y$ is not metaphysically definable from $x_{1} \ldots x_{n}$. Expanding out the definition of metaphysical definability, this is equivalent to the following principle: ${ }^{53}$

Fundamental Independence $\forall \bar{x} y \forall X(F u n(\bar{x} y) \wedge \operatorname{Pure}(X) \rightarrow \neg X \bar{x}=y)$

As with the structural principles, Fundamental Independence falls out of or theory of logical possibility: it follows from Quantified Logical Necessity and the plausible assumption that there are at least two things in

relations are without falling afoul of the paradoxes of higher-order vagueness. I maintain that the fundamental things are precise, and reject (ii). But one could draw the opposite conclusion and allow that there are fundamental properties and relations that are vague, undermining the above argument against Fundamental Completeness. (Note the difference between (i) and (ii): (i) says the things falling under Fun ${ }^{\sigma}$ are precise, (ii) asserts that the property Fun ${ }^{\sigma}$ is itself precise. Only (i) is used in our argument against Fundamental Completeness.)

${ }^{51}$ We might formalize this as: Hydrogen $=\lambda z \exists x y(z=F u s e(x, y) \wedge \operatorname{Electron}(x) \wedge \operatorname{Proton}(y) \wedge$ Bound $(y, x))$.

${ }^{52}$ For a more recent discussion of the independence idea see Dorr and Hawthorne (2013).

${ }^{53}$ Here, as usual, Fundamental Independence is schematic in the types of $X, y$ and $\bar{x}$, and $F u n(\bar{x} y)$ is short for the claim that $x_{1} \ldots x_{n}, y$ are fundamental and distinct (when of the same type). 
the same type as $y$. I leave the argument in a footnote. ${ }^{54}$

To illustrate Fundamental Independence let's work through some examples from the metaphysics literature. An oft examined application of the independence idea involves the case of converse relations. ${ }^{55}$ It's natural to think that there are asymmetric fundamental relations - perhaps, the relation of being more massive than. According to the no-redundancy idea, we shouldn't take as fundamental more relations than we need to: thus it shouldn't also be the case that being less massive than, the converse of the aforementioned relation, is also fundamental. In general:

$$
\operatorname{Fun}(R) \wedge \operatorname{Fun}(S) \wedge \neg R=S \rightarrow \neg(R=\lambda x y . S y x)
$$

We can prove this either from Fundamental Independence, or Separated Structure and the plausible assumption that at least one pair of relations (fundamental or non-fundamental) are not each others converses. Here is the argument from Separated Structure; it is slightly technical, but it is representative of how arguments using these principles typically go, so we shall present it. Let us write $P$ for the combinator $\lambda X Y . Y$, which (by $\beta$-equivalence) ignores its first argument and maps the second argument to itself, and let us write $Q$ for the combinator $\lambda X Y \lambda x y . X y x$, which ignores its second argument and maps its first argument to its converse. Thus, if I apply $P$ to $R$ and then $S$ I just get $R(P R S=R)$ and if I apply $Q$ to $R$ and $S$ I just get the converse of $S(Q R S=\lambda x y . S y x)$. Since $P$ and $Q$ are pure, and $R$ and $S$ fundamental and distinct, if $R$ is the converse of $S$, i.e. $R=\lambda x y$.Syx, then $P R S=Q R S$ and so $P=Q$ by Separated Structure. But then for any pair of relations $T$ and $U, P T U=Q T U$, and thus for any two relations $T$ and $U, T$ is the converse of $U$. But it's not true that every relation is the converse of every other relation - the only way that could happen is if there were exactly one relation and it was symmetric (it's own converse).

Sometimes these and related observations are taken to cast doubt on the independence principle (see Sider (2011)). On the one hand, reality shouldn't make invidious distinctions: there is an obvious symmetry between less massive than and more massive than, and it would be strange for reality to make a choice about which one was fundamental. Of course, we don't have to reject Fundamental Independence. Dorr (2004) takes these sorts of considerations to indicate that all fundamental relations are symmetric — we'll say more about this shortly. Fine (2000), on the other hand, uses these considerations to motivate a theory of relations that don't have the order of the argument places built into them. In Bacon (forthcoming) and Bacon (2019) I have suggested an alternative theoretical framework which does away with the ideology of fundamentality in favour of the notion of a fundamental basis. According to this picture there might be multiple collections of properties and relations that individually play the role that fundamentality plays in this paper: there might

\footnotetext{
${ }^{54}$ Suppose, for contradiction, that $x_{1} \ldots x_{n}, y$ are all distinct and fundamental and that $y=X \bar{x}$ for some pure $X$. Then by the necessity of identity we may infer that $\square y=X \bar{x}$, and so by the left-to-right direction of Logical Necessity we have $\forall \bar{z} w w=X \bar{z}$. But this clearly cannot hold for all $w$ and $\bar{z}$ if there are at least two elements in the same type as $y$.

${ }^{55}$ See, e.g. Dorr (2004), Sider (2011), McSweeney (forthcoming).
} 
be some fundamental bases that contain more massive than, and other equally good bases that contain less massive than, although none will contain both on account of the fact that bases should consist of independent properties and relations, in the sense that they should not be metaphysically definable from one another. (The analogy with bases of vector spaces is instructive here: every vector in a vector space can be built out of the elements of a basis, by a unique logical operation (in this context, 'logical operation' means linear combination). Vectors spaces therefore stand to their bases as languages stand to the primitive constants, in the sense that analogues of Fundamental Completeness and Fundamental Independence hold. But unlike languages, vector spaces may stand in this relation to many sets of vectors: they can have multiple bases. ${ }^{56}$ The suggestion, then, is that reality might be more like a vector space than a language.) At any rate, there are many positions on this matter that preserve the independence of the fundamental in some form without authorizing invidious metaphysical distinctions.

What about fundamental symmetric relations? Pace Dorr (2004), we can use our theory to show that no fundamental relation is symmetric. The reason is a special case of our previous result: if $R$ and $S$ are distinct fundamental relations then they are not converses of one another. But we can show that even if $R$ and $S$ are identical fundamental relations they are not converses - i.e. no fundamental relation can be its own converse:

$$
\operatorname{Fun}(R) \rightarrow \neg(R=\lambda x y \cdot R y x) .
$$

If $R=\lambda x y \cdot R y x$, then $I R=C R$, where $I$ is identity operation mapping each relation to itself, and $C$ the converse operation mapping each relation to its converse (both can be defined in purely logical terms as $\lambda X . X$ and $\lambda X \lambda x \lambda y . X y x$ respectively). By Separated Structure if $I R=C R$ then $I=C$. But if the identity and converse mappings on relations were identical, every relation would be identical to its converse; a hypothesis refuted by the existence of non-symmetric relations, like taller than.

Thus we have proved that no fundamental relation is symmetric. This conflicts with another piece of orthodoxy (Sider (2011), McSweeney (forthcoming)): that a logical operation like conjunction, for example, is fundamental and symmetric. I agree with the latter claim. ${ }^{57}$ But once we have properly embraced the present framework, in which there are two theoretically distinctive properties an entity can have fundamentality and purity - it's extremely natural to classify conjunction in the latter category.

This opens up wider questions about the status of our other theoretical primitives. We have argued that

\footnotetext{
${ }^{56}$ Indeed, we will show how to make this analogy formally precise in the next section. A basis $B$ for $V$ has the property that every function from $B$ to $V$ extends to a unique homomorphism (i.e. a linear transformation) of $V$. The constants $\Sigma$ of a language $\mathcal{L}$ have the property that any function from $\Sigma$ to $\mathcal{L}$ extends to a unique homomorphism (i.e. substitution) on $\mathcal{L}$. In the next section we will show how to formulate the analogous property for models of type theory.

${ }^{57}$ The symmetry of conjunction follows from Booleanism - the principle that Boolean equivalent propositions are identical (see footnote 20) - and the principle of Modalized Functionality, discussed earlier. By Booleanism, necessarily, for all $p$ and $q$, $\wedge p q=\wedge q p=(C \wedge) p q$, and so by Modalized Functionality we can conclude that $\wedge=C \wedge$.
} 
logical necessity is pure on the grounds that we have identified it with something defined in logical terms, $\lambda p \cdot p=\top$ (the broadest necessity). But what about the primitives of purity and fundamentality? Is the property of being pure itself pure?

Purity of Pure Pure ${ }^{\sigma \rightarrow t}\left(\right.$ Pure $\left.^{\sigma}\right)$

Or as Sider (2011) wonders, is the property of being fundamental itself fundamental?

Fundamentality of Fundamental $F u n^{\sigma \rightarrow t}\left(F u n^{\sigma}\right)$

Other options are possible as well: purity might be fundamental, fundamentality might be pure, and so on. Indeed, fundamentality or purity might themselves be neither fundamental nor pure. For instance, Sider considers the view, which he calls Melianism ${ }^{58}$, that being a fundamental property (say, of type $e \rightarrow t$ ) is just the disjunction of being identical to $F_{1}$ or $F_{2}$ or $\ldots$ or $F_{n}$, where $F_{1} \ldots F_{n}$ enumerate all the fundamental properties. This is a theory in which fundamentality can be metaphysically defined from the fundamental — in particular, from the properties $F_{1} \ldots F_{n}$ — via identity and disjunction, but is not itself fundamental or pure.

Some headway on the first question can be made if we adopt the analogue of Melianism for purity: that Pure $^{\sigma}$ is just the disjunction of being identical to $a_{1}$, or $\ldots$ or $a_{n}$ where $a_{1} \ldots a_{n}$ enumerate the pure elements of type $\sigma$, i.e. $\lambda x\left(x=a_{1} \vee \ldots \vee x=a_{n}\right)$. (If it is infinite a further assumption stating the existence of an infinite disjunction of properties is needed. ${ }^{59}$ ) Since identity, disjunction, and by assumption $a_{1} \ldots a_{n}$ are pure, it follows that Pure ${ }^{\sigma}$ is defined from pure things, and is itself pure (by Purity and Pure Application). ${ }^{60}$ On the other hand, paradoxes await if we assume that both purity and fundamentality are pure: one can define limited forms of substitution on propositions, allowing one to derive analogues of Gödel's diagonal lemma, and consequently propositional variants of the liar paradox. ${ }^{61}$

\footnotetext{
${ }^{58}$ Which he attributes to Joseph Melia.

${ }^{59}$ It takes the form of a completeness principle for properties, stating that any collection $X$ of properties (possibly infinite) has a disjunction: a property which is entailed by each property in $X$, and moreover is the weakest such property - it entails any other property that entails each property in $X$. A collection of properties of type $\sigma \rightarrow t$ can itself be represented by a property $X$ of type $(\sigma \rightarrow t) \rightarrow t$. A property, $F$, entails another, $G$, just in case $\square \forall_{\sigma} x(F x \rightarrow G x)$.

${ }^{60}$ Thanks to Cian Dorr for suggesting this argument to me. Note that even without assuming Melianism about purity, this argument establishes that Pure ${ }^{\sigma}$ is at minimum coextensive with a pure property: the Melian one. Of all the properties that have as their extension the pure entities, the pure Melian one seems to be a conspicuous choice to be the property of being pure. (Of course, we should take this argument with a pinch of salt since for all we've said there might be other pure, or even fundamental properties coextensive with it that would also make good candidates).

${ }^{61}$ For instance, one can define a diagonal property, $D$, stating of a proposition $p$ that the result of substituting $p$ for $p$ s only propositional fundamental constituent in $p$ is false: $\lambda p . \forall X r\left(\operatorname{Pure}^{t \rightarrow t}(X) \wedge F u n^{t}(r) \wedge p=X r \rightarrow \neg X p\right)$ (when $p$ doesn't have a single propositional fundamental constituent the statement is vacuously true). If Fun and Pure are pure, D is itself pure, and if, moreover, there was a fundamental proposition, $r$, then $D r$ would have exactly one fundamental propositional constituent, and $D(D r)$ would not be vacuously true. Indeed, using broadly Gödel-Tarski style reasoning, one can see that $D(D r)$ is true iff it isn't, appealing only to Quantified Separated Structure. Related issues are discussed in Bacon (forthcoming) (remarks 6 and 7), and Jeremy Goodman has an argument that generalizes these conclusions, showing there can't be fundamental entities in arbitrary types.
} 


\section{$5 \quad$ Metaphysical Substitutions}

So far we have been content to reason in the object language from our theory of logical possibility. In this section I will outline, in rough sketch, a model that unifies the main ideas we have discussed. Not only is the model theory used to prove the consistency of the theory, but it is heuristically valuable. The model theory gives us a simple way to characterize the three main concepts of the theory - logical possibility, fundamentality, and purity - and moreover illuminates the connections between them. Having a concrete model in sight also gives us a clearer sense of which sentences of higher-order logic might be worth investigating. This section is designed to guide the reader through some formal machinery that was devised to model the concepts employed in the preceding discussion, the result is a very broad overview of some technical material that is developed more rigorously elsewhere. ${ }^{62}$

Section 1 introduced a Bolzano-inspired account of logical truth in which a logically true sentence is a sentence with only true substitution instances. The central device in our model is that of a metaphysical substitution, which, putting it somewhat glibly, stands to reality (or at least, a given model of reality) as a substitution stands to language. That is, given a model of propositions, properties relations, and so forth, which designates some elements of the model as fundamental, a metaphysical substitution can be understood informally as determining a mapping taking each fundamental element to an arbitrary element of the same type. A metaphysical substitution can also be applied to non-fundamental elements of the model: informally we should think of them as replacing the fundamental constituents in those elements in accordance with the mapping.

Let's make this a little more precise. A model will determine, among other things, a domain, $D^{\sigma}$, of entities of type $\sigma$ for each type $\sigma$. $D^{t}$ represents the set of propositions, $D^{e \rightarrow t}$ the properties, $D^{t \rightarrow t}$ the operators, and so on. Since type $\sigma \rightarrow \tau$ corresponds to expressions that take an argument of type $\sigma$ and produce an expression of type $\tau$, it's natural to model elements of $D^{\sigma \rightarrow \tau}$ by a set of functions from $D^{\sigma}$ to $D^{\tau}$ (but not necessarily the set of all such functions). Since propositions can be true or false, a model will also determine a distinguished subset of $D^{t}$ corresponding to the propositions which are true.

Rather than assume directly that the elements of $D^{\sigma}$ have constituents to which one can apply substitutions, it is crucial to our approach that we adopt an abstract characterization of metaphysical substitutions. That is, we assume a set of abstract substitutions, $i, j, k, \ldots$, subject to some minimal laws: for example, that there be a trivial substitution 1 that leaves everything alone, and given any two substitutions $i, j$ there is another, $i \circ j$, which is the result of performing one after the other. We also adopt as primitive the notion of performing a substitution to an element of $D^{\sigma}$ : that for any element $a$ in any domain $D^{\sigma}$, there is another

\footnotetext{
${ }^{62}$ The reader should consult the appendix and Bacon (forthcoming) for further details.
} 
element $i a$ in $D^{\sigma}$ representing the result of performing the substitution $i$ to $a$. The notion of substitution performance is also subject to some minimal laws, the most important of which is that if $f \in D^{\sigma \rightarrow \tau}$ and $a \in D^{\sigma}$, then $i(f a)=(i f)(i a)$. Informally, if you apply a (possibly complex) predicate, $F$, to a (possibly complex) argument, $a$, and perform a substitution on the result, that's the same as performing the substitution directly on $F$ and on $a$, and then applying the result of the former to the latter. Full statements of the laws can be found in the appendix. ${ }^{63}$

Our definitions are guided, of course, by the structure in which $D^{\sigma}$ literally consists of closed expressions, of type $\sigma$, in a higher-order language, with the non-logical constants playing the role of the fundamental constituents. The abstract substitutions may then be thought of actual substitutions on that language functions from constants to closed expressions of matching type - and the notion of applying a substitution, $i$, to an expression $M \in D^{\sigma}$ is simply the result of replacing all occurrences of constants occurring in $M$ with the expressions $i$ maps them to, denoted $i M$. This structure clearly satisfies the laws outlined - for instance $i(F a)=(i F)(i a)$. But the definitions are sufficiently general that they can be applied even when the elements of $D^{\sigma}$ are not overtly structured, as the terms of a language are.

We'll use the notion of a metaphysical substitution to elucidate and connect three notions that we have been concerned with: logical possibility, fundamentality, and purity.

Let's start with the notion of logical possibility. Heeding the Bolzanian definition of logical truth, we might hope to find an interpretation of higher-order logic in which a proposition, $p \in D^{t}$, is a logical necessity iff, for every metaphysical substitution, $i$, ip is true (i.e. in the designated subset of $D^{t}$ ), and a logical possibility iff, for some metaphysical substitution, $i, i p$ is true. ${ }^{64}$ (Observe that our complaint regarding the Bolzanian definition of logical truth — that it is objectionably language relative — is circumvented entirely given the metaphysical, as opposed to linguistic, interpretation of the substitutions.)

The analogy in the above with possible world semantics is suggestive: substitutions stand to logical possibility as possible worlds stand to metaphysical possibility. Using that as our paradigm, it seems fitting to identify propositions with sets of substitutions, and the truth functional operations like conjunction and negation with the set-theoretic operations of intersection and complementation, and so forth.

A remark on this modeling choice is in order. The choice ensures Booleanism: the view that propositions are governed by the Boolean identities — equations, like $p \wedge(q \vee r)=(p \wedge q) \vee(p \wedge r)$, where both sides are equivalent in the propositional calculus. This is a view that is often thought to be antithetical to the structural view of propositions, for if Boolean equivalent propositions are identified, we cannot straightforwardly keep

\footnotetext{
${ }^{63}$ The general framework is also discussed in Bacon (forthcoming).

${ }^{64}$ This is a condition on models which can be made a bit more precise as follows. We may suppose that logical necessity is represented by an operator $N e c \in D^{t \rightarrow t}$, and is therefore a function mapping propositions to propositions. Thus the constraint amounts to the claim that $N e c(p)$ is in the distinguished set of true propositions (a subset of $D^{t}$ determined by a model) if and only if $i p$ is in that set for every substitution $i$. Dual things may be said about logical possibility.
} 
track of things like the number of times a constituent occurs — for example, $p$ and $p \vee p$ are Boolean equivalent and thus identical — or the order of the constituents, as, for instance, $p \vee q$ and $q \vee p$ are also identified. This might strike one as surprising given the structural principles we have been able to recover in the present theory, such as Separated Structure. Or indeed, the principle Logical Necessity, which endeavors to capture the idea of a proposition being true in virtue of its logical form; the notion of a logical form appears to be inherently structural.

It turns out that, despite appearances, all of the above principles are entirely consistent with Booleanism; a good-making feature, in my book, since this is a simple and well-understood theory of propositional granularity. The main difficulty with identifying propositions with sets of substitutions is that it is not clear what it means to apply a substitution to an unstructured set. For unlike a sentence or a structured proposition, we cannot 'look inside' an unstructured set of indices to find the simple constituents and replace them with other things. This is where the abstract approach to metaphysical substitutions pays off.

The problem is this: given a set of substitutions, $p$, and a substitution $i$, we want to know which set $i p$ is. The answer I have adopted is this: $i p$ is identified with the set of substitutions $j$ such that $j \circ i \in p$. This answer makes everything run smoothly, but it is not pulled entirely out of the hat either. (Readers willing to accept a quick moral — that this definition is not pulled from a hat, but is quite natural - may skip the rest of this paragraph.) We can derive it from the following postulate, inspired by the possible worlds theory. For if we look at the analogy between the two analyses of possibility, we find that a proposition being true under a substitution plays the same role that a proposition being true at a world plays in the possible worlds theory. In the possible worlds theory a proposition, qua set of worlds, is true at a world iff that world belongs to the proposition. Thus we postulate that the substitution of $p$, ip, is true just in case $p$ is true at $i$, adopting the same convention of saying that a set $p$ is true at $i$ iff $i \in p$ (recall here that a model determines a distinguished subset of $D^{t}$ as the true propositions): ${ }^{65}$

$i p$ is true if and only if $p$ is true at $i$

Several things following from this postulate. First, even if we do not know how to apply an arbitrary substitution to a proposition, we do know how to apply the trivial substitution: $1 p=p$. Thus $p$ is true simpliciter iff $1 p$ is true simpliciter, iff $p$ is true at 1 , i.e. iff $1 \in p$. Thus we have settled what the designated subset of $D^{t}$ corresponding to the truths must be: those propositions containing the trivial substitution, 1. Applying this to the indented principle we have that $1 \in i p$ if and only if $i \in p$. By multiplying both sides by an arbitrary substitution $j$, it follows straightforwardly that, in general, $j \in i p$ iff $j \circ i \in p$. ${ }^{66}$ But this

\footnotetext{
65 'truth' in what follows always refers to truth in a particular model where $D^{t}$ consists of sets of substitutions.

${ }^{66}$ The idea that we can 'multiply both sides by $j$ ' needs justification: $j \in i p$ iff $1 \in j(i p)$, applying our latest conclusion (that $j \in p$ iff $1 \in j p)$ to $i p$ instead of $p$. The right hand side is equivalent to $1 \in(j \circ i) p$, given how we are understanding $\circ$, and so by the same principle we conclude that this is equivalent to $j \circ i \in p$.
} 
biconditional tells us exactly which substitutions belong to $i p$ : the $j$ such that $j \circ i \in p$, as required.

Let us turn to the concept of fundamentality. A model also determines a subset of each domain, Fun ${ }^{\sigma}$, representing the elements of $D^{\sigma}$ that are fundamental. ${ }^{67}$ We will draw on the formal properties of constants in higher-order languages to impose analogous constraints on the fundamental. These will help secure principles, like Fundamental Independence and Fundamental Completeness from section 4, that capture the sense in which the fundamental are like primitive constants of a language. The distinctive property of constants, as opposed to complex expressions, in a language is that they freely generate the language. Informally this means that every expression of the language can be built out of the constants (via the term forming operations), and no unforced identifications between two expressions are made. This idea is ubiquitous in mathematics, where one talks about freely generated groups, vector spaces, rings, Boolean algebras, and countless other algebraic objects. In each case, the algebraic structure is generated by a set of 'fundamental' elements if every element of the algebra can be constructed out of those elements via the algebraic operations, and no two formal combinations of those elements are identified unless the algebraic laws dictate that they must.

Mathematicians have developed a beautiful and compelling account of this phenomenon - universal algebra - that systematizes the abstract structure common to all these examples by looking at when and how functions between the relevant algebras can be extended to homomorphisms. In the context of languages the notion of free generation amounts to the following. The set of constants freely generate the language because any function that takes each constant of the language to a closed expression of the same type can be extended to a unique substitution of the language. ${ }^{68}$ No set containing a complex expression, $M$, can have the above property, for there are functions on the set which cannot be extended to a substitution. This is because every substitution must map $M$ to an expression with the same logical structure, but not every function does this (except when $M$ is a constant, and it has no logical structure). ${ }^{69}$ Any set with this property must also contain all the constants, for otherwise there could be functions that could be extended to multiple substitutions that disagreed about what happens to the constants not included in the set.

We thus see that the existence of a substitution extending any given function ensures that the constants are not defined out of simpler things, and that the uniqueness ensures that our list of constants is complete. These two ideas correspond naturally to the idea that the fundamental are independent and cannot be

\footnotetext{
${ }^{67}$ Strictly speaking, the model provides us with a function $f u n^{\sigma} \in D^{\sigma \rightarrow t}$ - intuitively the property of being a fundamental entity of type $\sigma$ - which determines an extension as follows: Fun ${ }^{\sigma}$ is the set of $a \in D^{\sigma}$ such that $f u n^{\sigma}(a) \in D^{t}$ is true.

${ }^{68}$ Such definitions are sometimes formulated relative to a class of languages: that every function from the constants to expressions of another language in the class extends to a unique translation (see Lambek and Scott (1988), for example). Here we are considering a singleton class of languages, where a translation from a language to itself is just a substitution; when we talk about other algebraic structures we shall do the same. The loss of generality is not that important for our purposes, but it makes a substitutional (as opposed to translational) analysis of various concepts possible.

${ }^{69}$ Here is a concrete example: every substitution of $(p \wedge q)$, where $p$ and $q$ are propositional constants, must be of the form $(A \wedge B)$ for sentences $A$ and $B$. But evidently there are functions that map $(p \wedge q)$ to something not of this form, such as $\neg r$.
} 
defined out of simpler things, and the idea that everything can be defined by the fundamental. Indeed, the principles Fundamental Independence and Fundamental Completeness discussed in section 4 can be secured by analogous conditions in a model of the sort described above:

Fundamental Independence Any type-indexed collection of functions $f^{\sigma}: F u n^{\sigma} \rightarrow D^{\sigma}$ can be extended to at least one metaphysical substitution.

Fundamental Completeness Any type-indexed collection of functions $f^{\sigma}: F u n^{\sigma} \rightarrow D^{\sigma}$ can be extended to at most one metaphysical substitution.

For a type-indexed collection of functions $f^{\sigma}$ to extend to a substitution $i$ I simply mean that for every type $\sigma$ and $a \in F u n^{\sigma}, f^{\sigma}(a)=i a$.

Let us finally turn to the notion of a pure property. As I understand the notion, pure entities are constituentless operations, of which there is no straightforward analogue in language. Paradigm examples of such things are the combinators: operations denoted by expressions that are built just out of variables and $\lambda$, such as $\lambda x . x$. An approximate analogue of pure elements in language are expressions that do not contain any non-logical constants. ${ }^{70}$ Such expressions are left alone by substitutions (as there is nothing to substitute), which brings us to our final analysis:

Purity An element $a \in D^{\sigma}$ is pure if and only if $i a=a$ for every substitution $i$.

From this definition one can derive some desirable properties. For example, purity is closed under application: if you apply a pure operation to a pure argument the result is pure. ${ }^{71}$ And any element of $D^{\sigma}$ denoted by a combinator expression is pure. ${ }^{72}$

The above provides connected analyses of our three main concepts - logical possibility, fundamentality and purity - and furthers our running analogy between language and reality. Putting these ideas together, let me end by indicating, in big picture terms, how they might combine to secure the truth of Logical Necessity. For suppose that $f \in D^{\sigma_{1} \rightarrow \ldots \sigma_{n} \rightarrow t}$ is pure and takes fundamental arguments $a_{1} \ldots a_{n}$ (of types $\left.\sigma_{1} \ldots \sigma_{n}\right)$, all distinct, to a proposition, $f a_{1} \ldots a_{n}$. Employing our substitutional analyses of logical necessity, we see that this proposition is logically necessary in the model if and only if every substitution of it, $i\left(f a_{1} \ldots a_{n}\right)=(i f)\left(i a_{1}\right) \ldots\left(i a_{n}\right)$ is true. Every substitution fixes $f$ because it is pure, and moreover, assuming Fundamental Independence, there is a substitution taking $a_{1} \ldots a_{n}$ to any given $n$ tuple of elements

\footnotetext{
${ }^{70}$ This is only an approximate analogy. It is servicable because the logical constants are typically stipulated to be left alone by substitutions. But this analogy makes it seem like we have a choice in the matter of what operations in reality get fixed by a metaphysical substitution, and that one could define a wider class of substitutions that move the pure operations around if one wanted. This is not how it is: the identity operation $\lambda x . x$ has to be fixed by every metaphysical substitution (as must any combinator expression: see Bacon (forthcoming) proposition 4). For example $(\lambda x . x) p$ just is p: one couldn't substitute $\lambda x . x$ for some other operator, leaving $p$ alone, because the result of applying another operator to $p$ needn't be $p$.

${ }^{71}$ Formally, if $f \in D^{\sigma \rightarrow \tau}$ and $a \in D^{\sigma}$ are pure then $f a$ is pure. For any given substitution $i(f a)$ is $(i f)(i a)$. By the purity of $f$ and $a, i f=f$, and $i a=a$, so $i(f a)=f a$. Since $i$ was arbitrary, $f a$ is pure.

${ }^{72}$ For a proof of this fact see Bacon (forthcoming) proposition 4.
} 
matching the domains of $a_{1} \ldots a_{n}$. Thus we can see that $(i f)\left(i a_{1}\right) \ldots\left(i a_{n}\right)$ is true for every substitution $i$ if and only if $f$ is satisfied by every $n$-tuple of elements of the domain, in accordance with Logical Necessity. ${ }^{73}$

\section{Conclusion}

In this paper I have put forward a theory of logical necessity that vindicates the Humean idea that the fundamental properties and relations are freely recombinable. This theory not only characterizes the size of modal space but, I have argued, substantiates the purported metaphor of the fundamental as the constants of 'God's language of reality'.

The theory has some unintuitive features. It embraces the idea of logically contingent distinctness, and our model vindicates the thesis that propositions are individuated relatively coarsely, by Boolean equivalence. But we have also seen that the language-reality metaphor is treacherous and fraught with paradoxes: some compromises are to be expected. The Boolean theory is a conspicuous alternative to a straightforwardly structural theory and we have shown that it is consistent with many insights of the structural picture that do not on their own lead to inconsistency. I thus would like to recommend the present framework as an alternative for metaphysicians inclined to theorize about the fundamental in structural terms.

\section{References}

D. M. Armstrong. A Combinatorial Theory of Possibility. Cambridge University Press, 1989.

Andrew Bacon. The broadest necessity. Journal of Philosophical Logic, 47(5):733-783, 2018a.

Andrew Bacon. Vagueness and Thought. Oxford University Press, 2018b.

Andrew Bacon. Is reality fundamentally qualitative? Philosophical Studies, 176(1):259-295, 2019. doi: 10.1007/s11098-017-1015-1.

Andrew Bacon. Substitution structures. Journal of Philosophical Logic, pages 1-59, forthcoming. doi: 10.1007/s10992-019-09505-z.

Andrew Bacon, John Hawthorne, and Gabriel Uzquiano. Higher-order free logic and the Prior-Kaplan paradox. Canadian Journal of Philosophy, 46(4-5):493-541, 2016.

Cian Dorr. Non-symmetric relations. Oxford Studies in Metaphysics, 1:155-92, 2004.

\footnotetext{
${ }^{73}$ Notice that the condition for Fundamental Completeness is not used in this argument.
} 
Cian Dorr. There are no abstract objects. In Theodore Sider, John Hawthorne, and Dean W. Zimmerman, editors, Contemporary Debates in Metaphysics. Blackwell, 2008.

Cian Dorr. To be F is to be G. Philosophical Perspectives, 30(1):39-134, 2016.

Cian Dorr and John Hawthorne. Naturalness. In Karen Bennett and Dean Zimmerman, editors, Oxford Studies in Metaphysics: Volume 8, page 1. Oxford University Press, 2013.

John Etchemendy. The Concept of Logical Consequence. Harvard University Press, 1990.

Solomon Feferman. Which quantifiers are logical? In Alessandro Torza, editor, Quantifiers, Quantifiers, and Quantifiers: Themes in Logic, Metaphysics, and Language. Springer, 2015.

Hartry Field. Saving Truth From Paradox. Oxford University Press, 2008.

Kit Fine. The problem of de re modality. In John Perry, J. Almog, and Howard K. Wettstein, editors, Themes From Kaplan, pages 197-272. Oxford University Press, 1989.

Kit Fine. Neutral relations. Philosophical Review, 109(1):1-33, 2000.

Kit Fine. A theory of truthmaker content i: Conjunction, disjunction and negation. Journal of Philosophical Logic, 46(6):625-674, 2017.

Frederic B. Fitch. Symbolic Logic. New York: Ronald Press Co., 1952.

Peter Fritz. A purely recombinatorial puzzle. Nô̂s, 51(3):547-564, 2017. doi: 10.1111/nous.12172.

Jeremy Goodman. Reality is not structured. Analysis, 77(1):43-53, 2017.

Volker Halbach. The substitutional analysis of logical consequence. Nô̂s, forthcoming.

Harold T. Hodes. Why ramify? Notre Dame Journal of Formal Logic, 56(2):379-415, 2015.

Lloyd Humberstone. What Fa says about a. Dialectica, 54(1):3-28, 2000.

G. Kreisel. On weak completeness of intuitionistic predicate logic. Journal of Symbolic Logic, 27(2):139-158, 1962.

Saul Kripke. Naming and necessity. In Darragh Byrne and Max Kölbel, editors, Philosophy, pages 431-433. Routledge, 1980.

Saul A. Kripke. Outline of a theory of truth. Journal of Philosophy, 72(19):690-716, 1975. 
Joachim Lambek and Philip J Scott. Introduction to higher-order categorical logic, volume 7. Cambridge University Press, 1988.

David K. Lewis. On the Plurality of Worlds. Wiley-Blackwell, 1986.

Michaela Markham McSweeney. Following logical realism where it leads. Philosophical Studies, pages 1-23, forthcoming.

John Myhill. Problems arising in the formalization of intensional logic. Logique Et Analyse, 1(1):78-83, 1958.

Graham Priest. In Contradiction: A Study of the Transconsistent. Oxford University Press, 2006.

Arthur N. Prior. Formal Logic. Oxford, Clarendon Press, 1962.

Agustin Rayo and Gabriel Uzquiano. Toward a theory of second-order consequence. Notre Dame Journal of Formal Logic, 40(3):315-325, 1999.

Agustín Rayo and Timothy Williamson. A completeness theorem for unrestricted first-order languages. In Jc Beall, editor, Liars and Heaps. Oxford University Press, 2003.

Bertrand Russell. Mathematical logic as based on the theory of types. American Journal of Mathematics, 30(3):222-262, 1908.

Bertrand Russell. Principles of Mathematics. Routledge, 1937.

Jeffrey Sanford Russell and John Hawthorne. Possible patterns. Oxford Studies in Metaphysics, forthcoming.

J. Schaffer. The internal relatedness of all things. Mind, 119(474):341-376, 2010.

Stephen Schiffer. Vague properties. In Richard Dietz and Sebastiano Moruzzi, editors, Cuts and Clouds: Vagueness, its Nature, and its Logic, pages 109-130. Oxford University Press, 2010.

Stewart Shapiro. Foundations Without Foundationalism: A Case for Second-Order Logic. Oxford University Press, 1991.

Gila Sher. The Bounds of Logic: A Generalized Viewpoint. MIT Press, 1991.

Theodore Sider. Writing the Book of the World. Oxford University Press, 2011.

Alfred Tarski. Logic, Semantics, Metamathematics Papers From 1923 to 1938. Hackett, 1983.

Alfred Tarski. What are logical notions? History and Philosophy of Logic, 7(2):143-154, 1986. 
Dustin Tucker and Richmond H. Thomason. Paradoxes of intensionality. Review of Symbolic Logic, 4(3): 394-411, 2011.

Gabriel Uzquiano. A neglected resolution of russell's paradox of propositions. Review of Symbolic Logic, 8 (2):328-344, 2015.

Jennifer Wang. Fundamentality and modal freedom. Philosophical Perspectives, 30(1):397-418, 2016.

Timothy Williamson. Existence and contingency. Proceedings of the Aristotelian Society, 100(1):117-139, 2000.

Timothy Williamson. Everything. Philosophical Perspectives, 17(1):415-465, 2003.

Timothy Williamson. Modal Logic as Metaphysics. Oxford University Press, 2013.

Timothy Williamson. Modal science. Canadian Journal of Philosophy, 46(4-5):453-492, 2016. doi: 10.1080/00455091.2016.1205851.

Jessica M. Wilson. What is hume's dictum, and why believe it? Philosophy and Phenomenological Research, 80(3):595-637, 2010.

Ludwig Wittgenstein. Tractatus Logico-Philosophicus (Trans. Pears and McGuinness). Routledge, 1961. 


\section{Appendix}

\subsection{Higher-order logic}

The simple types consist of two base types, $e$ and $t$, and a function type $(\sigma \rightarrow \tau)$ whenever $\sigma$ and $\tau$ are simple types. A signature consists of a set $\Sigma$ of constants that have been assigned types. We write $\Sigma^{\sigma}$ for the set of constants of type $\sigma$. For each type $\sigma$ we also help ourselves to an infinite set of variables $\operatorname{Var}^{\sigma}$; we write $\operatorname{Var}$ for the set of variables of any type.

The terms over a signature $\Sigma$ are simultaneously constructed and assigned types inductively as follows:

1. $M$ is a term of type $\sigma$ if $M \in \Sigma^{\sigma} \cup \operatorname{Var}^{\sigma}$

2. (MN) is a term of type $\tau$ if $M$ has type $\sigma \rightarrow \tau$ and $N$ type $\sigma$

3. $\lambda x . M$ is a term of type $\sigma \rightarrow \tau$ if $x \in \operatorname{Var}^{\sigma}$ and $M$ a term of type $\tau$.

We will be solely concerned with higher-order languages in what follows. A higher-order language contains a logical constant $\forall_{\sigma}$ of type $(\sigma \rightarrow t) \rightarrow t$ for each $\sigma$ and a logical constant $\rightarrow$ of type $t \rightarrow t \rightarrow t$. Since all the signatures we will consider contain the logical constants, we write $\mathcal{L}(\Sigma)$ for the set of all terms over a signature $\Sigma$ of non-logical constants and $\mathcal{L}^{\sigma}(\Sigma)$ for the terms of type $\sigma . \mathcal{L}(\emptyset)$ is thus the purely logical language, whose only constants are the logical constants.

To improve readability we introduce a number of abbreviations. $\perp$ stands for $\forall_{t \rightarrow t} \forall_{t}, \top$ for $\rightarrow \perp \perp$,

$\neg$ for $\lambda p .(\rightarrow p) \perp, \wedge$ stands for $\lambda p \lambda q \neg(\rightarrow p(\neg q)), \vee$ for $\lambda p .(\rightarrow(\neg p)), \leftrightarrow$ for $\lambda p q(\wedge(\rightarrow p q)(\rightarrow q p)),={ }_{\sigma}$ for $\lambda x \lambda y \forall_{\sigma \rightarrow t} X(\rightarrow(X x)(X y))$ and $\square$ for $\left(=_{t} \top\right)$ and $\diamond$ for $\lambda p \neg \square \neg p$. We also follow the convention of writing binary defined operations in infix position; e.g. writing $p \rightarrow q$ instead of $(\rightarrow p) q$, and $\top=_{t} p$ instead of $\left(={ }_{t} \top\right) p$.

A theory over a language $\mathcal{L}(\Sigma)$ is a subset of $\mathcal{L}^{t}(\Sigma)$. A standard way to present a theory is via a collection of axioms and rules. Here is a minimal theory of higher-order logic, which we call $\mathrm{H}$ :

PC All instances of propositional tautologies.

MP From $A$ and $A \rightarrow B$ infer $B$

Gen From $A \rightarrow B$ infer $A \rightarrow \forall_{\sigma} x B$ when $x$ does not occur free in $A$.

UI $\forall_{\sigma} x A \rightarrow A[t / x]$ (where $t$ is a term of type $\sigma$ and no variable in $t$ gets bound when substituted into $A$ )

$\beta \eta A \leftrightarrow B$ whenever $A$ and $B$ are $\beta \eta$ equivalent terms of type $t$. 
A propositional tautology is simply any term of type $t$ with the form of a tautology. $\mathrm{H}$ is therefore the smallest set that contains every instance of a tautology, UI, and $\beta \eta$, and is closed under the MP and Gen rules. In the text we also assumed a further axiom and rule:

Rule of Equivalence From $A \leftrightarrow B$ infer $A={ }_{t} B$

Functionality $\forall_{\sigma \rightarrow \tau} X Y\left(\forall_{\sigma} x(X x=Y x) \rightarrow X=Y\right)$

We call the resulting system HFE. The Rule of Equivalence ensures the assumption of Booleanism: for if $A$ and $B$ are tautological equivalents then $A \leftrightarrow B$ is a member of $\mathrm{H}$ (and thus HFE).

\subsection{Surjective $M$-set models}

In this section we'll outline a class of structures that are sound with respect to our system HFE. Proofs of most of the following propositions and theorems (including the aforementioned soundness theorem) are suppressed for brevity.

Definition 7.1 (Surjective $M$-set). An $M$-set $(M, A, \mu)$ consists of a monoid $M$ and a set $A$ and an operation $\mu: M \times A \rightarrow A$ such that:

$$
\begin{aligned}
& \mu(1, a)=a \text { for all } a \in A \\
& \mu(i, \mu(j, a))=\mu(i \circ j, a) \text { for all } i, j \in M \text { and } a \in A
\end{aligned}
$$

$\mu$ is called the action of $M$ on $A$. An $M$-set is surjective iff

For an $i \in M, \mu(i, \cdot): A \rightarrow A$ is surjective: for every $a \in A$ there is an $a^{\prime} \in A$ such that $\mu\left(i, a^{\prime}\right)=a$.

We adopt the standard convention of suppressing $\mu$ and writing $i a$ as short for $\mu(i, a)$. When $M$ and $\mu$ are fixed in the context, we also suppress reference to them in $(M, A, \mu)$ and refer to the $M$-set by its set component, $A$.

In what follows we focus on surjective $M$-sets as these bear a special relationship to functional models of type theory: structures where the domain of type $\sigma \rightarrow \tau, A^{\sigma \rightarrow \tau}$, can be represented by a set of functions from $A^{\sigma}$ to $A^{\tau}$. Given two surjective $M$-sets $A$ and $B$ we can form another surjective $M$-set, written $A \Rightarrow B$, which we shall call the function space $M$-set. ${ }^{74}$

Definition 7.2 (Function space). If $A$ and $B$ are surjective $M$-sets then the function space $M$-set ( $M, A \Rightarrow$ $B, \mu)$ is defined:

\footnotetext{
${ }^{74}$ For those familiar with category theory, the category of surjective $M$-sets (whose arrows $f: A \rightarrow B$ are functions such that $f(i a)=i f(a)$ for every $i \in M)$ is cartesian closed, which $A \Rightarrow B$ playing the role of the exponential object and the usual product of $M$-sets the product.
} 


$$
\begin{aligned}
& A \Rightarrow B:=\left\{f: A \rightarrow B \mid \text { for any } i \in M, a, a^{\prime} \in A, \text { if } i a=i a^{\prime} \text { then } i(f a)=i\left(f a^{\prime}\right)\right\} \\
& \mu(i, f)=a \mapsto i(f b) \text { where } b \text { is any element of } A \text { such that } i b=a .
\end{aligned}
$$

Note that $\mu(i, f)$ is well-defined provided $f \in A \Rightarrow B$ : if $i b=a$ and $i b^{\prime}=a$ then $i(f b)=i\left(f b^{\prime}\right)$ by the condition for belonging to $A \Rightarrow B$. Moreover, we know that there is at least one $b$ such that $i b=a$ since $A$ is a surjective $M$-set.

Proposition 8. If $A$ and $B$ are surjective $M$-sets then $A \Rightarrow B$ is a surjective $M$-set.

The proof is mostly straightforward: we include the proof of surjectivity in a footnote as it is illustrative. ${ }^{75}$ Finally we show how to construct a model of higher-order logic from these notions.

Definition 8.1 (Full surjective $M$-set model). A $\Sigma$-model over a monoid $M$ consists of a triple $\left(A, \llbracket \cdot \rrbracket^{`}\right)$ where $A$ is an applicative structure and:

1. $A^{e}$ is a surjective $M$-set

2. $A^{t}$ is the surjective $M$-set $P(M)$ with the action of division: $p \in A^{t}$, ip $=\{j \in M \mid j \circ i \in p\}$

3. $A^{\sigma \rightarrow \tau}:=A^{\sigma} \Rightarrow A^{\tau}$

4. $\operatorname{App}^{\sigma \tau}(f, a)=f(a)$

5. $\llbracket \cdot \rrbracket^{\sigma}: \Sigma^{\sigma} \rightarrow A^{\sigma}$ is a type indexed collection of interpretation functions such that:

(a) $\llbracket \rightarrow \rrbracket^{t \rightarrow t \rightarrow t}(p)(q)=(M \backslash p) \cup q$

(b) $\llbracket \forall_{\sigma} \rrbracket^{(\sigma \rightarrow t) \rightarrow t}(f)=\bigcap_{a \in A^{\sigma}} f a$

Borrowing terminology from modal logic, we call an $\emptyset$-model $a$ frame, and a $\Sigma$-model with the same first and last component a model over that frame.

$A$ element $p \in A^{t}$ is a proposition, and we say that it's true iff $1 \in p$.

It is easily seen that with the provided actions the applicative structure underlying a $\Sigma$-model forms a substitution structure in the sense of Bacon (forthcoming). Specifically, the action on the function space is defined by stipulating that $(i f)(i a)=i(f b)$ where $b$ is any element where $i b=i a$. By choosing $b:=a$ we can infer that $(i f)(i a)=i(f a)$, as required of a substitution structure. The other conditions on substitution structures are satisfied trivially.

\footnotetext{
${ }^{75}$ If $i \in M$ and $g \in A \Rightarrow B$ let us set $f(a):=b$ where $b \in B$ is any element such that $i b=g(i a)$. Since $B$ is surjective we know that there is such an element $b$. In general there will be multiple choices of $b$, but however we make these choices the resulting function always belongs to $A \Rightarrow B$ : if $i a=i a^{\prime}$ then $i(f a)=g(i a)$ and $i\left(f a^{\prime}\right)=g\left(i a^{\prime}\right)$. But since $g \in A \Rightarrow B$ it follows that $g(i a)=g\left(i a^{\prime}\right)$, and so $i(f a)=i\left(f a^{\prime}\right)$ as required.

Lastly note that $(i f)=g$. Let $a \in A$. Then $(i f) a=i\left(f a^{\prime}\right)$ for some $a^{\prime} \in A$ with $i a^{\prime}=a . f\left(a^{\prime}\right)=b$ where $i b=g\left(i a^{\prime}\right)$; so $i\left(f a^{\prime}\right)=g\left(i a^{\prime}\right)$. Finally $g\left(i a^{\prime}\right)=g(a)$ since $i a^{\prime}=a$. Thus (if) $a=g a$ for every $a \in A$ and so $i f=g$.
} 
It is straightforward to check that $\llbracket \rightarrow \rrbracket^{t \rightarrow t \rightarrow t}$ and $\llbracket \forall_{\sigma} \rrbracket^{(\sigma \rightarrow t) \rightarrow t}$ really do belong to $A^{t \rightarrow t \rightarrow t}$ and $A^{(\sigma \rightarrow t) \rightarrow t}$ respectively. We will follow the convention of referring to models and frames by their first component (i.e. $A$ instead of $(A, \llbracket \rrbracket))$. If we are working in the $t$ fragment of the type heirarchy (types built out of $t$ s only) then the full surjective $M$-set model is uniquely determined by $M$, and we write it $A(M)$. Otherwise it is uniquely determined by $M$ and the choice of $A^{e}$. We omit the superscripts from $\llbracket \rrbracket$ when no ambiguity arises.

A variable assignment is a function taking, for each type $\sigma$, each element of $\operatorname{Var}^{\sigma}$ to $A^{\sigma}$. $\llbracket \cdot \rrbracket$ can be extended to an mapping from arbitrary terms and variable assignments in the usual way. That is:

$$
\begin{aligned}
& \llbracket c \rrbracket^{g}=\llbracket c \rrbracket \text { for } c \in \Sigma \\
& \llbracket x \rrbracket^{g}=g(x) \text { for } x \in V a r \\
& \llbracket M N \rrbracket^{g}=A p p\left(\llbracket M \rrbracket^{g}, \llbracket N \rrbracket^{g}\right) . \\
& \llbracket \lambda x \cdot M \rrbracket^{g}=a \mapsto \llbracket M \rrbracket^{g[x \mapsto a]}
\end{aligned}
$$

In the last clause it must be shown that the relevant function indeed belongs to $A^{\sigma \rightarrow \tau}$. A closed term of type $t, A$, is true in $A$ iff $\llbracket A \rrbracket$ is true (i.e. $1 \in \llbracket A \rrbracket)$. If $A$ is a frame we write $T h^{\Sigma}(A)$ for the logic of a frame over the signature $\Sigma$ :

$T h^{\Sigma}(A):=\left\{C \in \mathcal{L}(\Sigma) \mid C\right.$ is true in $A^{\prime}$ for every $\Sigma$-model $A^{\prime}$ over the frame $\left.A\right\}$.

If $\mathcal{C}$ is a class of frames we similarly write $T h^{\Sigma}(\mathcal{C})$ for $\bigcap_{A \in \mathcal{C}} T h^{\Sigma}(A)$.

Here are two useful facts about $\Sigma$-models over a monoid $M$.

Proposition 9. The interpretation of $\forall_{\sigma}$ and $\rightarrow$ are pure in any $\Sigma$-model: $i \llbracket \forall_{\sigma} \rrbracket^{(\sigma \rightarrow t) \rightarrow t}=\llbracket \forall_{\sigma} \rrbracket^{(\sigma \rightarrow t) \rightarrow t}$ for every $i \in M$ and similarly for $\rightarrow$.

Proposition 10. If $\phi$ is a closed term of type $t, A$ a frame (over $M$ ), and $i \in M$, then:

$$
i \in \llbracket \square \phi \rrbracket \text { iff, for every } j \in M, j \circ i \in \llbracket \phi \rrbracket \text {. }
$$

In particular, from the definition of the action on $A^{t}$ we know that: $\square \phi \rrbracket$ is true iff, for every $i \in M$, $i \llbracket \phi \rrbracket$ is true. ${ }^{76}$ This corresponds to the substitutional analysis of logical necessity, in which a proposition is necessary iff all of its metaphysical substitutions are true (compare with Bolzano's substitutional analysis of logical truth, in which a sentence is true iff all of its substitution instances are).

\footnotetext{
${ }^{76}$ Note that for any $p \in A^{t}$ and $i, j \in M, j \in i p$ iff $j \circ i \in p$. Thus $\llbracket \square \phi \rrbracket$ is true iff $1 \in \llbracket \square \phi \rrbracket$, iff $j \circ 1=1 \circ j \in \llbracket \phi \rrbracket$ for every $j$ (by proposition 10), iff $1 \in j \llbracket \phi \rrbracket$ for every $j$. So $\llbracket \square \phi \rrbracket$ is true (contains 1 ) iff, for every $j \in M, j \llbracket \phi \rrbracket$ is true
} 
If we stipulate that a term of type $t$ is true at a substitution $i$ iff $i \in \llbracket \phi \rrbracket$, then proposition 10 takes the form of a familiar clause for modal operators in which $\square \phi$ is true at a world iff $\phi$ is true at every world accessible to it. It is therefore sometimes helpful to think of $M$ as a Kripke frame:

Definition 10.1. Let $M$ be a monoid. The induced rooted Kripke frame, $\mathcal{F}(M)=\left(W, \leq, w_{0}\right)$, is defined as follows:

1. $W:=M$

2. $i \leq j$ iff there is a $k \in M$ such that $k \circ i=j$.

3. $w_{0}:=1$

Via this connection, the model described here can equivalently be seen as an instance of the model theory based on modalized domains described in the appendix of Bacon (2018a).

\subsection{A model of Logical Necessity}

Here we sketch in brief a proof of the consistency of Logical Necessity and Individuation relative to a signature $\Sigma$ of non-logical constants representing the fundamental entities. We then show how one can interpret Fun and Pure in the model in order to validate Quantified Logical Necessity. We begin by restricting attention to the type $t$ fragment of the type hierarchy as this illustrates the idea of the proof in its purest form. The full details of the argument (which includes type $e$ ) will be spelled out in future work.

Let $M$ be the monoid:

$$
\begin{aligned}
& M:=\mathbb{N}^{<\omega} \text {, finite sequences of natural numbers. } \\
& 1=\langle\rangle, \text { the empty sequence. } \\
& i \circ j:=i j, \text { the concatenation of the two sequences. }
\end{aligned}
$$

It is also helpful to think of the induced Kripke frame which we shall denote by $\mathcal{H}$. The worlds of this frame are finite sequences of natural numbers, and accessibility the relation of being an initial segment of. The the frame thus has a tree structure in which every node has infinitely many children: ${ }^{77}$

\footnotetext{
${ }^{77}$ Thanks to Catrin Campbell-Moore for creating this diagram.
} 


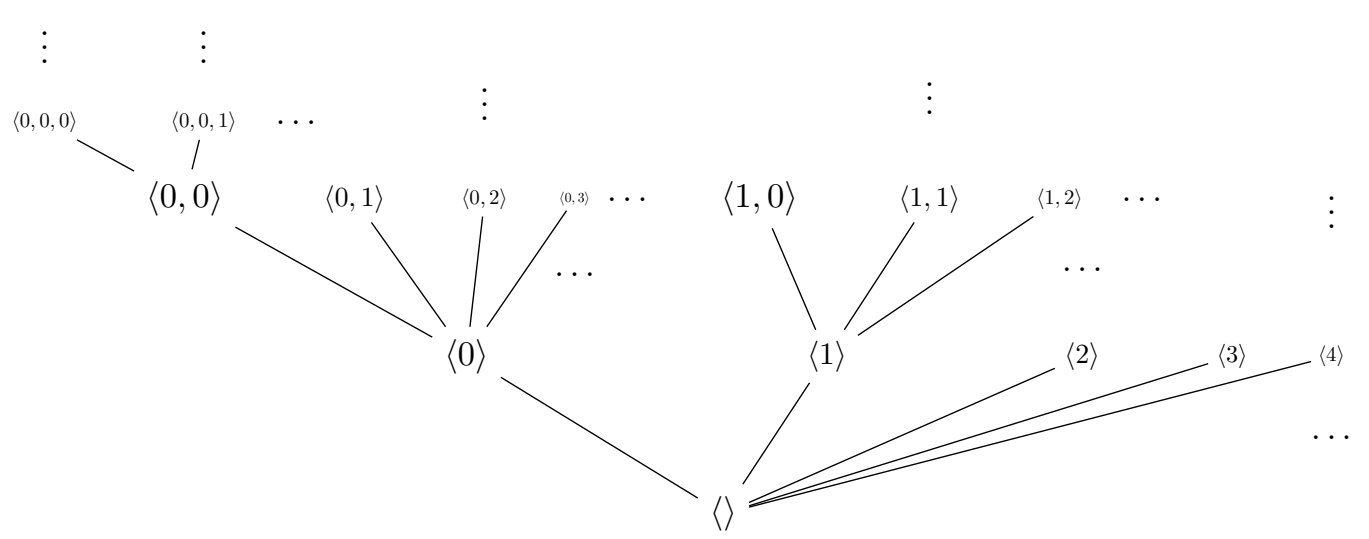

What is the action on $P(M)$ (i.e. $\left.A^{t}(M)\right)$ in the frame $A(M)$ ? For $i \in M$ and $p \in P(M)$, we may picture $p$ as a subregion of the infinite $V$ shape that is our tree ( $\mathcal{H}$ depicted above). The worlds that live above $i$ form an identical $V$ shape, and $p$ also carves a subregion of those: we may think of $i p$ as the result of pulling the shape $p$ makes in the $V$ above $i$ down to the root of the tree (thus overwriting whatever shape $p$ had outside of the $V$ sprouting from $i$ ). Every substitution $i$ also has a right inverse defined as: $i^{-1} p=\{i j \mid j \in p\} . i^{-1}$ can be thought of shifting a shape upwards from the shape it occupies at the root to the shape it occupies at $i$.

The consistency proof rests on two basic ideas. Let $A(M)$ be the frame over $M . A(M)$ is a model of pure higher-order logic, i.e. a model of the language $\mathcal{L}(\emptyset)$. Firstly note that the following three things are equivalent for a sentence $\phi \in \mathcal{L}(\Sigma)$.

1. $\phi$ is consistent with $T h^{\Sigma}(A(M))$ (i.e. its negation is not a member).

2. $\phi$ is true in $A^{\prime}$ for some $\Sigma$-model $A^{\prime}$ over $A(M)$.

3. $\exists \bar{x} \phi[\bar{x} / \bar{c}]$ is true in $A(M)$.

4. $\exists \bar{x} \phi[\bar{x} / \bar{c}]$ is true in $A^{\prime}$ for any $\Sigma$-model $A^{\prime}$ over $A(M)$.

The first two are equivalent by definition. 2 and 3 are equivalent: a witness of for the formula $\exists \bar{x} \phi[\bar{x} / \bar{c}]$ in $A(M)$ - i.e. a sequence $\bar{a}$ of elements of $A(M)$ that satisfy $\phi[\bar{x} / \bar{c}]$ in this model — provide the means to extend the interpretation of $A(M)$ to $\Sigma$ in a way that makes $\phi(\bar{c})$ true. Namely interpret $c_{i}$ by $a_{i}$. Similarly an interpretation of $c_{1} \ldots c_{n}$ that makes $\phi(\bar{c})$ true in $A(M)$ provides a witness for $\phi[\bar{x} / \bar{c}]$. Finally 3 and 4 are equivalent because $\exists \bar{x} \phi[\bar{x} / \bar{c}]$ contains only logical vocabulary and $A$ and $A(M)$ agree about the interpretations of logical vocabulary.

The next part of the proof appeals to the following theorem:

Theorem 10.1. Suppose that $A_{1}, A_{2}, A_{3} \ldots$ are a $\Sigma$-models over the frame $A(M)$, with interpretation functions $\llbracket \cdot \rrbracket_{1}, \llbracket \cdot \rrbracket_{2}, \llbracket \cdot \rrbracket_{3} \ldots$ Then there is a $\Sigma$-model $A$ over $A(M)$ such that: 
1. $\langle n\rangle \llbracket c \rrbracket=\llbracket c \rrbracket_{n}$ for every $n \in \mathbb{N}$ and $c \in \Sigma$

2. $\langle n\rangle \llbracket \alpha \rrbracket=\llbracket \alpha \rrbracket_{n}$ for every $n \in \mathbb{N}$ and arbitrary terms $\alpha \in \mathcal{L}(\Sigma)$

Of course, part (2) follows from (1) by a routine induction using the fact that $\llbracket \forall_{\sigma} \rrbracket$ and $\llbracket \rightarrow \rrbracket$ are fixed by every substitution. To illustrate the theorem, suppose that the signature $\Sigma$ contains only propositional letters. For a propositional letter $p$, it is easy to see how to construct an interpretation $\llbracket p \rrbracket \subseteq W$ with $\langle n\rangle \llbracket p \rrbracket=\llbracket p \rrbracket_{n}$ for each $n \in \mathbb{N}$ : simply let $\llbracket p \rrbracket=\bigcup_{n}\left(\langle n\rangle^{-1} \llbracket p \rrbracket_{n}\right)$. (Note that we could add 1 to $\left.\llbracket p \rrbracket\right)$. It's clear that the result of shifting $\llbracket p \rrbracket$ down from $\langle n\rangle$ to the root \langle\rangle will result in $\llbracket p \rrbracket_{n}$. Theorem 10.1 generalizes this idea to arbitrary signatures which might include non-propositional types. We do not have the space to prove it here, however it follows from a more general construction, that I will describe elsewhere, for gluing together models of the sort described in Bacon (2018a).

The second part of the proof can now proceed. For a given signature $\Sigma$ we enumerate the $\mathcal{L}(\Sigma)$ sentences consistent in the logic $T h^{\Sigma}(A(M)): \phi_{1}, \phi_{2}, \phi_{3}, \ldots$, and enumerate a collection of $\Sigma$-models $A_{1}, A_{2}, A_{3} \ldots$ over $A(M)$ such that $A_{n} \models \phi_{n}$. Using theorem 10.1 we may construct a global model $A$ and note that the following are equivalent

1. $\phi$ is consistent in $T h^{\Sigma}(A(M)$

5. $\diamond \phi$ is true in $A$

If $\phi$ is consistent with $T h^{\Sigma}\left(A(M)\right.$ then for some $n, \phi=\phi_{n}$ and is true in $A_{n}$ : i.e. $1 \in \llbracket \phi \rrbracket_{n}$. By theorem 10.1, $1 \in\langle n\langle\llbracket \phi \rrbracket$. By the definition of the action of $\langle n\rangle$ this means that $\langle n\rangle \in \llbracket \phi \rrbracket$ and so by proposition 10 $1 \in \llbracket \diamond \phi \rrbracket$; i.e. $\diamond \phi$ is true in $A$. Conversely, if $\diamond \phi$ is true in $A-$ i.e. $1 \in \llbracket \diamond \phi \rrbracket$ then by proposition 10 , $i \in \llbracket \phi \rrbracket$ for some $i \in M$. We may define another $\Sigma$-model $A_{i}$ over $A(M)$ by setting $\llbracket c \rrbracket_{i}=i \llbracket c \rrbracket$ for each $c \in \Sigma$. A straightforward induction on terms reveals that $1 \in \llbracket \chi \rrbracket_{i}$ iff $i \in \llbracket \chi \rrbracket$. So in particular $\phi$ is true in $A_{i}$, and thus is $T h^{\Sigma}(A(M))$-consistent.

In order to validate Quantified Logical Necessity we also need to interpret the predicates Pure and Fun. This can be achieved by augmenting As interpretation function as follows.

- $\llbracket P u r e_{\sigma} \rrbracket^{\sigma \rightarrow t}(a)=\{i \mid i a=\llbracket \alpha \rrbracket$ for some purely logical closed term $\alpha \in \mathcal{L}(\emptyset)\}$.

- $\llbracket F u n_{\sigma} \rrbracket^{\sigma \rightarrow t}(a)=\{i \mid i a=\llbracket c \rrbracket$ for some $c \in \Sigma\}$.

It may be shown that the resulting interpretation validates Quantified Logical Necessity. 